\title{
SZEGÕ CONDITION AND SCATTERING FOR ONE-DIMENSIONAL DIRAC OPERATORS
}

\author{
R. V. BESSONOV
}

\begin{abstract}
We prove existence of modified wave operators for one-dimensional Dirac operators whose spectral measures belong to the Szegó class on the real line.
\end{abstract}

\section{INTRODUCTION}

Consider the one-dimensional Dirac operator $\mathcal{D}_{Q}$ on the half-axis $\mathbb{R}_{+}=[0,+\infty)$,

$$
\mathcal{D}_{Q}: X \mapsto J X^{\prime}+Q X, \quad J=\left(\begin{array}{cc}
0 & -1 \\
1 & 0
\end{array}\right), \quad Q=\left(\begin{array}{cc}
q_{1} & q_{2} \\
q_{2} & -q_{1}
\end{array}\right) .
$$

We assume that functions $q_{1}, q_{2}$ are real and absolutely integrable on compact subsets of $\mathbb{R}_{+}$. The "free" Dirac operator with potential $Q=0$ will be denoted by $\mathcal{D}_{0}$. Let $L^{2}\left(\mathbb{R}_{+}, \mathbb{C}^{2}\right)$ be the Hilbert space of measurable functions $X: \mathbb{R}_{+} \rightarrow \mathbb{C}^{2}$ such that $\int_{\mathbb{R}_{+}}\|X(t)\|_{\mathbb{C}^{2}}^{2} d t<\infty$. The operator $\mathcal{D}_{Q}$ defined by (1) on absolutely continuous functions $X \in L^{2}\left(\mathbb{R}_{+}, \mathbb{C}^{2}\right)$ such that $\mathcal{D}_{Q} X \in L^{2}\left(\mathbb{R}_{+}, \mathbb{C}^{2}\right),\left\langle X(0),\left(\begin{array}{l}0 \\ 1\end{array}\right)\right\rangle_{\mathbb{C}^{2}}=0$, is the self-adjoint operator on $L^{2}\left(\mathbb{R}_{+}, \mathbb{C}^{2}\right)$, see Section 8.6 in [10]. The standard wave operators for the pair $\mathcal{D}_{0}, \mathcal{D}_{Q}$ are defined as the limits

$$
W_{ \pm}\left(\mathcal{D}_{Q}, \mathcal{D}_{0}\right)=\lim _{t \rightarrow \mp \infty} e^{i t \mathcal{D}_{Q}} e^{-i t \mathcal{D}_{0}}
$$

in the strong operator topology in the case where these limits exist. Given a potential $Q=\left(\begin{array}{cc}q_{1} & q_{2} \\ q_{2} & -q_{1}\end{array}\right)$, we write $Q \in L^{p}$ if $q_{1}, q_{2} \in L^{p}\left(\mathbb{R}_{+}\right)$. The classical scattering theory implies the existence of wave operators $W_{ \pm}\left(\mathcal{D}_{Q}, \mathcal{D}_{0}\right)$ for $Q \in L^{1}$, see, e.g., Theorem XI.9 in [11]. In 2002, Christ and Kiselev [2] proved the existence of wave operators $W_{ \pm}\left(\mathcal{D}_{Q}, \mathcal{D}_{0}\right)$ for Dirac operators with potentials $Q \in L^{p}, 1 \leqslant p<2$. Using a different approach, Denisov [4] extended their result to the class $Q \in L^{2}$. His proof gives a formula for $W_{ \pm}\left(\mathcal{D}_{Q}, \mathcal{D}_{0}\right)$ in terms of the Szegó function of the spectral measure for $\mathcal{D}_{Q}$. A measure $\mu=w d x+\mu_{\mathbf{s}}$ on the real line $\mathbb{R}$ belongs to the Szegó class $\mathrm{Sz}(\mathbb{R})$ if $\left(1+x^{2}\right)^{-1} \in L^{1}(\mu)$ and

$$
\int_{\mathbb{R}} \frac{\log w(x)}{1+x^{2}} d x>-\infty
$$

where $w$ is the density of the absolutely continuous part of $\mu$. The Szegó function $D_{\mu}$ of $\mu \in \mathrm{Sz}(\mathbb{R})$ is the outer function in the upper half-plane $\mathbb{C}^{+}=\{z \in \mathbb{C}: \operatorname{Im} z>0\}$ with modulus $\sqrt{w}$ on $\mathbb{R}$ and such that $D_{\mu}(i)>0$. In other words,

$$
D_{\mu}(z)=\exp \left(\frac{1}{\pi i} \int_{\mathbb{R}} \log \sqrt{w(x)}\left(\frac{1}{x-z}-\frac{x}{x^{2}+1}\right) d x\right), \quad z \in \mathbb{C}^{+} .
$$

2010 Mathematics Subject Classification. 34L40, 42C05.

Key words and phrases. Dirac system, wave operators, Szegő class.

The author is supported by RFBR grant mol a dk 16-31-60053. 
Let $\mu$ be the spectral measure of the operator $\mathcal{D}_{Q}$, so that the generalized Fourier transform

$$
\mathcal{F}_{Q}: X \mapsto \frac{1}{\sqrt{\pi}} \int_{\mathbb{R}_{+}}\langle X(t), \Psi(t, \bar{z})\rangle_{\mathbb{C}^{2}} d t, \quad z \in \mathbb{C},
$$

densely defined on functions with compact support, is the isometric operator from $L^{2}\left(\mathbb{R}_{+}, \mathbb{C}\right)$ to $L^{2}(\mu)$. Here $\left\langle\left(\begin{array}{l}a_{1} \\ a_{2}\end{array}\right),\left(\begin{array}{l}b_{1} \\ b_{2}\end{array}\right)\right\rangle_{\mathbb{C}^{2}}=a_{1} \bar{b}_{1}+a_{2} \bar{b}_{2}$, and $\Psi$ denotes the generalized eigenvector of $\mathcal{D}_{Q}$ :

$$
J \frac{\partial}{\partial t} \Psi(t, z)+Q \Psi(t, z)=z \Psi(t, z), \quad \Psi(0, z)=\left(\begin{array}{c}
1 \\
0
\end{array}\right), \quad t \geqslant 0, \quad z \in \mathbb{C} .
$$

We choose the normalization in (44) so that the Lebesgue measure on $\mathbb{R}$ is the spectral measure for $\mathcal{D}_{0}$. It is known that every Dirac operator $\mathcal{D}_{Q}$ on $\mathbb{R}_{+}$with locally integrable potential $Q$ has the unique spectral measure. Moreover if $Q \in L^{2}$, then the spectral measure $\mu$ of $\mathcal{D}_{Q}$ belongs to the Szegó class $\mathrm{Sz}(\mathbb{R})$, see Theorem 12.1 and Theorem 14.4 in [5]. It was proved by Denisov [4] that for all $Q \in L^{2}$ the wave operators $W_{ \pm}\left(\mathcal{D}_{Q}, \mathcal{D}_{0}\right)$ exist and

$$
W_{-}\left(\mathcal{D}_{Q}, \mathcal{D}_{0}\right)=\gamma \mathcal{F}_{Q}^{-1} \chi_{E} D_{\mu}^{-1} \mathcal{F}_{0}, \quad W_{+}\left(\mathcal{D}_{Q}, \mathcal{D}_{0}\right)=\bar{\gamma} \mathcal{F}_{Q}^{-1} \chi_{E} \overline{D_{\mu}^{-1}} \mathcal{F}_{0},
$$

where $\chi_{E}$ is the indicator of a Borel set $E$ such that $|\mathbb{R} \backslash E|=0, \mu_{\mathbf{s}}(\mathbb{R} \backslash E)=0$. Here and in what follows $\mu_{\mathbf{s}}$ denotes the singular part of $\mu$. We also denoted by $\chi_{E} D_{\mu}^{-1}$ the multiplication operator from $L^{2}(\mathbb{R})$ to $L^{2}(\mu)$ taking $f$ into $\chi_{E} D_{\mu}^{-1} f$. The parameter $\gamma \in \mathbb{C}$ in (6) depends only on $Q,|\gamma|=1$. Moreover, $\gamma=1$ for potentials $Q$ with zero entries on the diagonal $\left(q_{1}=0\right)$. It is not difficult to see that the operator in (6) is unitary from $L^{2}\left(\mathbb{R}_{+}, \mathbb{C}^{2}\right)$ onto the absolutely continuous subspace of $\mathcal{D}_{Q}$. In other words, the operators $W_{ \pm}\left(\mathcal{D}_{Q}, \mathcal{D}_{0}\right)$ for $Q \in L^{2}$ are complete.

It is known that for every $p>2$ there exists a potential $Q \in L^{p}$ such that the absolutely continuous spectrum of $\mathcal{D}_{Q}$ is empty. In particular, the strong wave operators $W_{ \pm}\left(\mathcal{D}_{Q}, \mathcal{D}_{0}\right)$ for such potentials $Q$ do not exist. Thus, the result of Denisov is sharp in the scale of $L^{p}$-spaces. However, there are locally integrable potentials $Q \notin \cup_{1 \leqslant p \leqslant 2} L^{p}$ such that the spectral measures of operators $\mathcal{D}_{Q}$ belong to the Szegó class $\mathrm{Sz}(\mathbb{R})$. For such measures $\mu$ the Szegó function $D_{\mu}$ and the right hand side of (6) are well-defined. It is natural to expect that the limits in (21) defining wave operators $W_{ \pm}\left(\mathcal{D}_{Q}, \mathcal{D}_{0}\right)$ exist as well. Examples constructed by Teplyaev [13] show that this is not the case, see the discussion after Theorem 14.6 in [5]. In fact, the number $\gamma$ in (6) is the limit of a function $e^{i \varphi(t)}$ related to the solution of (5) at the point $z=i$. If $Q \notin L^{2}$, this function can have non-vanishing oscillations as $t \rightarrow \infty$. To handle these oscillations, one can introduce the modified wave operators

$$
W_{ \pm}^{m}\left(\mathcal{D}_{Q}, \mathcal{D}_{0}\right)=\lim _{t \rightarrow \mp \infty} e^{i t \mathcal{D}_{Q}} \Sigma_{\varphi} e^{-i t \mathcal{D}_{0}}, \quad \Sigma_{\varphi} X=\left(\begin{array}{c}
\cos \varphi \sin \varphi \\
-\sin \varphi \cos \varphi
\end{array}\right) X .
$$

Following Denisov's approach for $Q \in L^{2}$, we prove existence of $W_{ \pm}^{m}\left(\mathcal{D}_{Q}, \mathcal{D}_{0}\right)$ for general Dirac operators with spectral measures in Szegó class. This requires some new constructions and estimates inspired by the theory of orthogonal polynomials on the unit circle. Our main result can be summarized as follows.

Theorem 1. Let $q_{1}, q_{2}$ be real-valued functions on $\mathbb{R}_{+}$such that $q_{1}, q_{2} \in L^{1}[0, r]$ for every $r>0$, and let $Q=\left(\begin{array}{cc}q_{1} & q_{2} \\ q_{2} & -q_{1}\end{array}\right)$. Assume that the spectral measure $\mu$ of $\mathcal{D}_{Q}$ belongs to $\mathrm{Sz}(\mathbb{R})$. Then there exists a function $\varphi$ such that the strong wave operators $W_{ \pm}^{m}\left(\mathcal{D}_{Q}, \mathcal{D}_{0}\right)$ in (17) exist and complete. Moreover, $W_{ \pm}^{m}\left(\mathcal{D}_{Q}, \mathcal{D}_{0}\right)$ coincide with the 
operators in (6) for $\gamma=1$. If $q_{1}=0$, then $\varphi=0$ and the usual wave operators $W_{ \pm}\left(\mathcal{D}_{Q}, \mathcal{D}_{0}\right)$ exist and complete.

Applying results of [1], one can explicitly characterize potentials $Q=\left(\begin{array}{cc}0 & q \\ q & 0\end{array}\right)$ such that the spectral measure of $\mathcal{D}_{Q}$ belongs to the Szegó class. For $n \geqslant 0$, introduce the functions

$$
g_{n}(t)=\exp \left(2 \int_{n}^{t} q(s) d s\right), \quad t \in[n, n+2) .
$$

As we will see in Section [5, the spectral measure of $\mathcal{D}_{Q}$ belongs to $\mathrm{Sz}(\mathbb{R})$ if and only if

$$
\sum_{n \geqslant 0}\left(\int_{n}^{n+2} g_{n}(t) d t \cdot \int_{n}^{n+2} \frac{d t}{g_{n}(t)}-4\right)<\infty .
$$

Taylor expansion of the function $e^{x}$ at $x=0$ shows that if

$$
\sum_{n \geqslant 0} \max _{n \leqslant t \leqslant n+1}\left(\int_{n}^{t} q(s) d s\right)^{2}<\infty
$$

then relation (8) holds. If one replaces $q$ by $|q|$ in (9), then the resulting class of potentials will coincide with $\ell^{2}\left(L^{1}\left(\mathbb{R}_{+}\right)\right)$, the endpoint of the scale $\ell^{p}\left(L^{1}\left(\mathbb{R}_{+}\right)\right)$, $1 \leqslant p<2$, treated by Christ and Kiselev in 2. It is interesting to note that the class of potentials described by (9) contains some non-decaying potentials. For example, the function $q: x \mapsto \sin \left(x^{2}\right)$ satisfies (9) but does not belong to any class $L^{p}\left(\mathbb{R}_{+}\right)$or $\ell^{p}\left(L^{1}\left(\mathbb{R}_{+}\right)\right), 1 \leqslant p<\infty$. Theorem 1 implies the following result.

Corollary 1. Let $q$ be a function on $\mathbb{R}_{+}$such that $q \in L^{1}[0, r]$ for all $r>0$, and let $\mathcal{D}_{Q}$ be the Dirac operator on $L^{2}\left(\mathbb{R}_{+}, \mathbb{C}^{2}\right)$ with the potential $Q=\left(\begin{array}{ll}0 & q \\ q & 0\end{array}\right)$. If $q$ satisfies (8) or (91), then the wave operators $W^{ \pm}\left(\mathcal{D}_{Q}, \mathcal{D}_{0}\right)$ exist.

A part of our analysis is close to Khrushchev ideas in the theory of orthogonal polynomials on the unit circle. One of the central results of Khrushchev paper 8 concerns a logarithmic convergence of orthogonal polynomials, see Theorem 2.5 in [8]. Let us formulate its analogue for measures $\mu$ on the real line.

Theorem 2. Let $\mu=w d x+\mu_{\mathbf{s}}$ be a measure in the Szegó class $\mathrm{Sz}(\mathbb{R})$, and let $\left\{\mathcal{B}_{r}\right\}$ be the chain de Branges spaces isometrically embedded into $L^{2}(\mu)$. Then

$$
\lim _{r \rightarrow \infty} \int_{\mathbb{R}}\left|\log \frac{1}{\left|E_{r}(x)\right|^{2}}-\log w(x)\right| \frac{d x}{1+x^{2}}=0,
$$

for some Hermite-Biehler functions $E_{r}$ generating $\mathcal{B}_{r}$.

More details on Theorem 2 can be found in Section 3. Section 4 deals with the main instrument of the present paper - regularized Krein's orthogonal entire functions. In Section 5 we prove Theorem 1 Next section concerns the theory of canonical Hamiltonian systems that will be used in the paper.

\section{Canonical Hamiltonian systems}

In this section we collect some known facts related to the spectral theory of canonical Hamiltonian systems, discuss the definition and properties of an entropy function introduced in [1, recall the notion of de Branges chains, and reduce consideration of Dirac systems to canonical Hamiltonian systems. 
2.1. Canonical Hamiltonian systems. The canonical Hamiltonian system on the positive half-axis $\mathbb{R}_{+}=[0,+\infty)$ is the differential equation

$$
\left\{\begin{array}{l}
J \frac{\partial}{\partial t} M(t, z)=z \mathcal{H}(t) M(t, z), \quad t \in \mathbb{R}_{+}, \quad z \in \mathbb{C} . \\
M(0, z)=\left(\begin{array}{ll}
1 & 0 \\
0 & 1
\end{array}\right),
\end{array}\right.
$$

As before, $J=\left(\begin{array}{cc}0 & -1 \\ 1 & 0\end{array}\right)$ is the constant sign matrix, the Hamiltonian $\mathcal{H}$ is a matrixvalued mapping such that

$$
\mathcal{H}=\left(\begin{array}{cc}
h_{1} & h \\
h & h_{2}
\end{array}\right), \quad \text { trace } \mathcal{H}(t)>0, \quad \operatorname{det} \mathcal{H}(t) \geqslant 0, \quad t \in \mathbb{R}_{+} .
$$

The functions $h_{1}, h_{2}, h$ are assumed to be real-valued and belong to $L_{l o c}^{1}\left(\mathbb{R}_{+}\right)$. Here and below $L_{l o c}^{1}\left(\mathbb{R}_{+}\right)$denotes the set of functions $f$ on $\mathbb{R}_{+}$such that $f \in L^{1}[0, r]$ for every $r>0$. We will often represent the solution $M$ of (10) in the form

$$
M(t, z)=(\Theta(t, z), \Phi(t, z))=\left(\begin{array}{c}
\Theta^{+}(t, z) \Phi^{+}(t, z) \\
\Theta^{-}(t, z) \Phi^{-}(t, z)
\end{array}\right), \quad t \in \mathbb{R}_{+}, \quad z \in \mathbb{C} .
$$

Since $\mathcal{H}$ is locally integrable, the function $M$ is locally absolutely continuous with respect to $t$ if the spectral parameter $z \in \mathbb{C}$ is fixed. It is also easy to see that for every $t \in \mathbb{R}_{+}$the entries of $M$ are entire functions with respect to $z$. A Hamiltonian $\mathcal{H}$ on $\mathbb{R}_{+}$is called singular if

$$
\int_{0}^{+\infty} \operatorname{trace} \mathcal{H}(t) d t=+\infty .
$$

We say that $\mathcal{H}$ is trivial if $\mathcal{H}$ coincides with one of two matrices $\left(\begin{array}{ll}1 & 0 \\ 0 & 0\end{array}\right),\left(\begin{array}{ll}0 & 0 \\ 0 & 1\end{array}\right)$ almost everywhere on $\mathbb{R}_{+}$. Fix a parameter $\omega \in \mathbb{R} \cup\{\infty\}$. The Weyl function of $\mathcal{H}$ is defined by

$$
m(z)=\lim _{t \rightarrow+\infty} \frac{\omega \Phi^{+}(t, z)+\Phi^{-}(t, z)}{\omega \Theta^{+}(t, z)+\Theta^{-}(t, z)}, \quad z \in \mathbb{C}^{+},
$$

where $\mathbb{C}^{+}=\left\{z \in \mathbb{C}^{+}: \operatorname{Im} z>0\right\}$. It can be shown [6] that for every singular nontrivial Hamiltonian $\mathcal{H}$ the expression under the limit in (13) is correctly defined for large $t>0$ (the denominator is non-zero), it does not depend on $\omega$, and, moreover, $m$ is the analytic function in $\mathbb{C}^{+}$with strictly positive imaginary part. In particular, $m$ admits the Herglotz representation

$$
m(z)=\frac{1}{\pi} \int_{\mathbb{R}}\left(\frac{1}{x-z}-\frac{x}{x^{2}+1}\right) d \mu(x)+b z+a, \quad z \in \mathbb{C}^{+},
$$

where $\mu$ is a Radon measure on $\mathbb{R}$ such that $\int_{\mathbb{R}} \frac{d \mu(x)}{x^{2}+1}<\infty, b \geqslant 0$, and $a \in \mathbb{R}$. Krein - de Branges theorem [7], 3] says that any function with positive imaginary part is the Weyl function of a singular nontrivial Hamiltonian on $\mathbb{R}_{+}$, see also [14, [12. The measure $\mu$ in (14) is called the spectral measure of the Hamiltonian $\mathcal{H}$. We will say that $\mu$ generates a singular nontrivial Hamiltonian $\mathcal{H}$ on $\mathbb{R}_{+}$if the Weyl function $m$ of $\mathcal{H}$ satisfies (14) for $\mu$ and $a=b=0$. A $\sigma$-finite Borel measure $\mu$ on $\mathbb{R}_{+}$is called even if $\mu(I)=\mu(-I)$ for every interval $I$. It is well-known that even measures generate diagonal Hamiltonians, see, e.g., [1]. By the diagonal Hamiltonian $\operatorname{diag}\left(h_{1}, h_{2}\right)$ we mean the matrix-function $\left(\begin{array}{cc}h_{1} & 0 \\ 0 & h_{2}\end{array}\right)$ on $\mathbb{R}_{+}$.

The main result of [1] is the following Szegó-type theorem for diagonal canonical Hamiltonian systems. 
Theorem 3. Let $\mu$ be an even measure on $\mathbb{R}_{+}$such that $(1+x)^{-2} \in L^{1}(\mu)$, and let $\mathcal{H}=\operatorname{diag}\left(h_{1}, h_{2}\right)$ be a Hamiltonian generated by $\mu$. Then $\mu \in \mathrm{Sz}(\mathbb{R})$ if and only if $\sqrt{\operatorname{det} \mathcal{H}} \notin L^{1}\left(\mathbb{R}_{+}\right)$and

$$
\sum_{n=0}^{+\infty}\left(\int_{\eta_{n}}^{\eta_{n+2}} h_{1}(s) d s \cdot \int_{\eta_{n}}^{\eta_{n+2}} h_{2}(s) d s-4\right)<\infty
$$

where $\eta_{n}=\min \left\{t \geqslant 0: \int_{0}^{t} \sqrt{\operatorname{det} \mathcal{H}(s)} d s=n\right\}$ for $n \geqslant 0$.

As we will see in Section 2.4. Theorem 3 implies that the spectral measure of the Dirac operator $\mathcal{D}_{Q}$ with a potential $Q=\left(\begin{array}{ll}0 & q \\ q & 0\end{array}\right)$ belong to the Szegó class $\mathrm{Sz}(\mathbb{R})$ if and only if relation (8) holds.

2.2. Entropy function of a Hamiltonian. Let $\mathcal{H}$ be a singular nontrivial Hamiltonian on $\mathbb{R}_{+}$. For every $r \geqslant 0$ define $\mathcal{H}_{r}$ to be the Hamiltonian on $\mathbb{R}_{+}$taking $x$ into $\mathcal{H}(x+r)$. Let $m_{r}, \mu_{r}, b_{r}, a_{r}$ denote the Weyl function, the spectral measure, and the coefficients in Herglotz representation (14) for $m_{r}$. Define

$$
\begin{aligned}
\mathcal{I}_{\mathcal{H}}(r) & =\operatorname{Im} m_{r}(i)=\frac{1}{\pi} \int_{\mathbb{R}} \frac{d \mu_{r}(x)}{1+x^{2}}+b_{r}, \\
\mathcal{R}_{\mathcal{H}}(r) & =\operatorname{Re} m_{r}(i)=a_{r}, \\
\mathcal{J}_{\mathcal{H}}(r) & =\frac{1}{\pi} \int_{\mathbb{R}} \frac{\log w_{r}(x)}{1+x^{2}} d x,
\end{aligned}
$$

where $w_{r}$ is the density of the absolutely continuous part of $\mu_{r}=w_{r} d x+\mu_{r, \mathbf{s}}$. It can be shown that $\mathcal{R}_{\mathcal{H}}$ is identically zero if the Hamiltonian $\mathcal{H}$ is diagonal, see Lemma 2.2 in [1]. Following [1], define the entropy function of $\mathcal{H}$ by

$$
\mathcal{K}_{\mathcal{H}}(r)=\log \mathcal{I}_{\mathcal{H}}(r)-\mathcal{J}_{\mathcal{H}}(r), \quad r \geqslant 0 .
$$

Since $b_{r} \geqslant 0$ by construction, from Jensen inequality we see that $\mathcal{K}_{\mathcal{H}}(r) \geqslant 0$. Consider the Hamiltonian

$$
\widehat{\mathcal{H}}_{r}(t)= \begin{cases}\mathcal{H}(t), & t \in[0, r), \\
\left(\begin{array}{cc}
c_{1}(r) & c(r) \\
c(r) & c_{2}(r)
\end{array}\right), & t \in[r,+\infty),\end{cases}
$$

where $c_{1}(r)=1 / \mathcal{I}_{\mathcal{H}}(r), c(r)=\mathcal{R}_{\mathcal{H}}(r) / \mathcal{I}_{\mathcal{H}}(r), c_{2}(r)=\left(\mathcal{I}_{\mathcal{H}}^{2}(r)+\mathcal{R}_{\mathcal{H}}^{2}(r)\right) / \mathcal{I}_{\mathcal{H}}(r)$. The Hamiltonian $\widehat{\mathcal{H}}_{r}$ coincides with $\mathcal{H}$ on $[0, r)$, is constant on $[r,+\infty)$, and, moreover, we have $\mathcal{K}_{\widehat{\mathcal{H}}_{r}}(0) \leqslant \mathcal{K}_{\mathcal{H}}(0)$, see Appendix. We call $\widehat{\mathcal{H}}_{r}$ the Bernstein-Szegó approximation to $\mathcal{H}$. The following three results were proved in [1] for diagonal Hamiltonians, see Appendix for the general case.

Lemma 1. Let $\mathcal{H}$ be a singular nontrivial Hamiltonian on $\mathbb{R}_{+}$and let $\mu=w d x+\mu_{\mathbf{s}}$ be the spectral measure of $\mathcal{H}$. Assume that $\mu \in \mathrm{Sz}(\mathbb{R})$. Then for every $r \geqslant 0$ the measure $\mu_{r}=w_{r} d x+\mu_{r, \mathbf{s}}$ is in $\mathrm{Sz}(\mathbb{R})$ and we have

(a) $m(z)=\frac{G_{r}(z)}{F_{r}(z)}$ for all $z \in \mathbb{C}^{+}$,

(b) $w(x)=\frac{w_{r}(x)}{\left|F_{r}(x)\right|^{2}}$ for almost all $x \in \mathbb{R}$,

where $G_{r}: z \mapsto \Phi^{+}(r, z)+m_{r}(z) \Phi^{-}(r, z)$ and $F_{r}: z \mapsto \Theta^{+}(r, z)+m_{r}(z) \Theta^{-}(r, z)$.

Recall that functions $\Theta^{ \pm}, \Phi^{ \pm}$in Lemma 1 are defined in (11). 
Lemma 2. Let $\mathcal{H}$ be a singular nontrivial Hamiltonian on $\mathbb{R}_{+}$whose spectral measure belongs to the Szegó class $\mathrm{Sz}(\mathbb{R})$. Then $\mathcal{K}_{\mathcal{H}}$ is the non-negative non-increasing absolutely continuous function and we have

$$
\lim _{r \rightarrow+\infty} \mathcal{K}_{\mathcal{H}}(r)=0, \quad \lim _{r \rightarrow+\infty} \mathcal{K}_{\widehat{\mathcal{H}}_{r}}(0)=\mathcal{K}_{\mathcal{H}}(0) .
$$

Lemma 3. Let $\mathcal{H}=\left(\begin{array}{cc}h_{1} & h \\ h & h_{2}\end{array}\right)$ be a singular nontrivial Hamiltonian on $\mathbb{R}_{+}$whose spectral measure belongs to the Szegố class $\mathrm{Sz}(\mathbb{R})$. Then the functions $\mathcal{I}_{\mathcal{H}}, \mathcal{R}_{\mathcal{H}}, \mathcal{K}_{\mathcal{H}}$ are locally absolutely continuous and

$$
\begin{aligned}
\mathcal{K}_{\mathcal{H}}^{\prime} & =2 \sqrt{h_{1} h_{2}-h^{2}}-\mathcal{I}_{\mathcal{H}} h_{1}-\frac{h_{2}}{\mathcal{I}_{\mathcal{H}}}+\frac{2 \mathcal{R}_{\mathcal{H}} h}{\mathcal{I}_{\mathcal{H}}}-\frac{\mathcal{R}_{\mathcal{H}}^{2} h_{1}}{\mathcal{I}_{\mathcal{H}}}, \\
\frac{\mathcal{I}_{\mathcal{H}}^{\prime}}{\mathcal{I}_{\mathcal{H}}} & =\mathcal{I}_{\mathcal{H}} h_{1}-\frac{h_{2}}{\mathcal{I}_{\mathcal{H}}}+\frac{2 \mathcal{R}_{\mathcal{H}} h}{\mathcal{I}_{\mathcal{H}}}-\frac{\mathcal{R}_{\mathcal{H}}^{2} h_{1}}{\mathcal{I}_{\mathcal{H}}}, \\
\frac{\mathcal{R}_{\mathcal{H}}^{\prime}}{\mathcal{I}_{\mathcal{H}}} & =2 \mathcal{R}_{\mathcal{H}} h_{1}-2 h,
\end{aligned}
$$

almost everywhere on $\mathbb{R}_{+}$. We also have $\mathcal{K}_{\mathcal{H}}(t)=\mathcal{K}_{\mathcal{H}^{d}}(t)$ for all $t \geqslant 0$ and the dual Hamiltonian $\mathcal{H}^{d}=J^{*} \mathcal{H} J$.

2.3. De Branges chains. Let $\mathcal{H}$ be a singular nontrivial Hamiltonian on $\mathbb{R}_{+}$. Define the Hilbert space

$$
\begin{aligned}
L^{2}(\mathcal{H})= & \left\{X: \mathbb{R}_{+} \rightarrow \mathbb{C}^{2}: \int_{\mathbb{R}_{+}}\langle\mathcal{H}(t) X(t), X(t)\rangle_{\mathbb{C}^{2}} d t<\infty\right\} / \mathcal{K} \text { er } \mathcal{H}, \\
& \mathcal{K} \text { er } \mathcal{H}=\left\{X: \mathcal{H}(t) X(t)=0 \text { for almost all } t \in \mathbb{R}_{+}\right\} .
\end{aligned}
$$

As usual, the inner product in $L^{2}(\mathcal{H})$ is defined by

$$
(X, Y)_{L^{2}(\mathcal{H})}=\int_{\mathbb{R}_{+}}\langle\mathcal{H}(t) X(t), Y(t)\rangle_{\mathbb{C}^{2}} d t .
$$

An open interval $I \subset \mathbb{R}_{+}$is called indivisible for $\mathcal{H}$ if there exists a vector $e \in \mathbb{R}^{2}$ such that $\mathcal{H}$ coincides with the rank-one operator $f \mapsto\langle f, e\rangle_{\mathbb{C}^{2}} e$ almost everywhere on $I$, and $I$ is the maximal open interval (with respect to inclusion) having this property. Let $\Im(\mathcal{H})$ denote the set of all indivisible intervals of the Hamiltonian $\mathcal{H}$, and let $\mathcal{M}$ be the complement in $\mathbb{R}_{+} \cup\{+\infty\}$ of the union of all intervals $I \in \mathfrak{I}(\mathcal{H})$. For $r \in \mathcal{M}$, define the subspace

$$
H_{r}=\left\{X \in L^{2}(\mathcal{H}): \operatorname{supp} X \subset[0, r], X=x_{I} \text { on } I \cap[0, r), I \in \mathfrak{I}(\mathcal{H}), x_{I} \in \mathbb{C}^{2}\right\} .
$$

Let $\Theta$ be the first column of the matrix $M$ in (11). Recall that $\langle\cdot, \cdot\rangle_{\mathbb{C}^{2}}$ denotes the inner product in $\mathbb{C}^{2},\left\langle\left(\begin{array}{c}a_{1} \\ a_{2}\end{array}\right),\left(\begin{array}{l}b_{1} \\ b_{2}\end{array}\right)\right\rangle_{\mathbb{C}^{2}}=a_{1} \bar{b}_{1}+a_{2} \bar{b}_{2}$. Consider the mapping

$$
\mathcal{W}_{\mathcal{H}}: X \rightarrow \frac{1}{\sqrt{\pi}} \int_{\mathbb{R}_{+}}\langle\mathcal{H}(t) X(t), \Theta(t, \bar{z})\rangle_{\mathbb{C}^{2}} d t, \quad z \in \mathbb{C},
$$

densely defined on $H_{+\infty}$ on functions with compact support. It is clear that $\mathcal{W}_{\mathcal{H}} X$ is the entire function with respect to $z$. The spectral measure of $\mathcal{H}$ could be characterized as the unique measure $\mu$ on $\mathbb{R}$ such that $\left(1+x^{2}\right)^{-1} \in L^{1}(\mu)$ and the mapping $\mathcal{W}_{\mathcal{H}}$ is the isometric operator from $H_{+\infty}$ to $L^{2}(\mu)$. Uniqueness of the measure $\mu$ follows from the fact that the Hamiltonian is singular (relation (12) holds). More details can be found in Sections 8, 9 of [12] and in [14]. 
Given a nonzero measure $\mu$ on $\mathbb{R}$ with $\left(1+x^{2}\right)^{-1} \in L^{1}(\mu)$, we construct the de Branges chain associated to $\mu$ as follows. First, set $a=b=0$ and define $m$ by (14). Then use Krein-de-Branges theorem to construct a Hamiltonian $\mathcal{H}$ on $\mathbb{R}_{+}$ such that $m$ is the Weyl function for $\mathcal{H}$. Define subspaces $H_{r} \subset L^{2}(\mathcal{H})$ and the transform $\mathcal{W}_{\mathcal{H}}$ in (18). The de Branges chain associated to $\mu$ consists of subspaces

$$
\mathcal{B}_{r}=\mathcal{W}_{\mathcal{H}} H_{r}, \quad r \in \mathcal{M} .
$$

Note that the elements of $\mathcal{B}_{r}$ are entire functions. In fact, $\mathcal{B}_{r}$ is the Hilbert space equipped with the inner product inherited from $L^{2}(\mu)$. By construction, the subspaces $\mathcal{B}_{r}$ are isometrically embedded into $L^{2}(\mu)$. We also have isometric inclusions $\mathcal{B}_{r_{1}} \subset \mathcal{B}_{r_{2}}$ for $r_{1} \leqslant r_{2}, r_{1,2} \in \mathcal{M}$. Any regular de Branges space (a Hilbert space of entire functions having few natural properties) isometrically embedded into $L^{2}(\mu)$ coincides with one of spaces $\mathcal{B}_{r}$ in de Branges chain associated to $\mu$. This fact is a consequence of de Branges chain theorem, see Section 35 in [3]. In particular, it implies the uniqueness of de Branges chain of $\mu$.

In general, it is not known how to construct a nontrivial Hilbert space of entire functions isometrically embedded into $L^{2}(\mu)$ avoiding the usage of the inverse spectral theory for canonical Hamiltonian systems. However, for measures $\mu$ in the Szegô class $\mathrm{Sz}(\mathbb{R})$ the situation simplifies. The following proposition is well-known for specialists.

Proposition 1. Let $\mu \in \mathrm{Sz}(\mathbb{R})$, and let $\left(\mathrm{PW}_{s}, \mu\right)$ be the completion with respect to the inner product of $L^{2}(\mu)$ of the linear space $\mathcal{E}_{s}$ of functions with smooth Fourier transform supported on $(-s, s)$. Then for every $s>0$ the Hilbert space $\left(\mathrm{PW}_{s}, \mu\right)$ belongs to the de Branges chain $\left\{\mathcal{B}_{r}\right\}$ associated to $\mu$. We have $\left(\mathrm{PW}_{s}, \mu\right)=\mathcal{B}_{r}$, where $r=\inf \left\{t: s=\int_{0}^{t} \sqrt{\operatorname{det} \mathcal{H}(\tau)} d \tau\right\}$.

This result is a consequence of de Branges theorems 23, 35 in [3] and Krein formula for exponential type (Theorem 11 in [12]).

Subspaces in de Branges chains admit a useful description in terms of HermiteBiehler functions associated to canonical Hamiltonian systems. An entire function $E$ belongs to the Hermite-Biehler class if $|E(z)|>\left|E^{\sharp}(z)\right|$ for all $z \in \mathbb{C}^{+}$. Here and below we denote $f^{\sharp}(z)=\overline{f(\bar{z})}$ for an entire function $f$. Let $\mathcal{H}$ be a singular nontrivial Hamiltonian on $\mathbb{R}_{+}$and let $\mu$ be its spectral measure. We will say that a Hermite-Biehler function $E$ generates the subspace $\mathcal{B}_{r}$ of de Branges chain of $\mu$ if

$$
\mathcal{B}_{r}=\left\{\text { entire } f: \frac{f}{E} \in H^{2}\left(\mathbb{C}^{+}\right), \frac{f^{\sharp}}{E} \in H^{2}\left(\mathbb{C}^{+}\right)\right\},
$$

and, moreover, we have $\|f\|_{L^{2}(\mu)}=\|f\|_{L^{2}\left(|E|^{-2} d x\right)}$ for every $f \in \mathcal{B}_{r}$. As usual, $H^{2}\left(\mathbb{C}^{+}\right)$denotes the standard Hardy space in the upper half-plane $\mathbb{C}^{+}$. The standard choice for the Hermite-Biehler function generating $\mathcal{B}_{r}$ is the function

$$
E_{r}: z \mapsto \Theta^{+}(r, z)+i \Theta^{-}(r, z), \quad z \in \mathbb{C},
$$

where $\Theta^{ \pm}$are the entries of the matrix $M$ in (11), see Section 3 in [12. If $E$ is any Hermite-Biehler function generating $\mathcal{B}_{r}$, then it is easy to see that the function

$$
k_{\mathcal{B}_{r}, \lambda}: z \mapsto-\frac{1}{2 \pi i} \frac{E(z) \overline{E(\lambda)}-E^{\sharp}(z) \overline{E^{\sharp}(\lambda)}}{z-\bar{\lambda}}, \quad z \in \mathbb{C},
$$

is the reproducing kernel of $\mathcal{B}_{r}$. This means that $k_{\mathcal{B}_{r}, \lambda} \in \mathcal{B}_{r}$ and we have $f(\lambda)=$ $\left(f, k_{\mathcal{B}_{r}, \lambda}\right)_{L^{2}(\mu)}$ for every $f \in \mathcal{B}_{r}$. 
Given a measure $\mu \in \mathrm{Sz}(\mathbb{R})$ and a number $s>0$, denote by $\left(\mathrm{PW}_{[0,2 s]}, \mu\right)$ the set of functions $e^{i s z} f$, where $f \in\left(\mathrm{PW}_{s}, \mu\right)$. Note that $\left(\mathrm{PW}_{[0,2 s]}, \mu\right)$ is the Hilbert space of entire functions with respect to the $L^{2}(\mu)$-inner product. Let $D_{\mu}$ be the Szegó function (3) of $\mu$. Proposition 1 implies that $\left(\mathrm{PW}_{[0,2 s]}, \mu\right)$ can be identified with the subspace of the weighted Hardy space

$$
H^{2}(w)=\left\{h=f D_{\mu}^{-1}, \quad f \in H^{2}\left(\mathbb{C}^{+}\right)\right\}, \quad w=\left|D_{\mu}\right|^{2},
$$

where the norm is defined by

$$
\|h\|_{H^{2}(w)}^{2}=\int_{\mathbb{R}}|h(x)|^{2} w(x) d x .
$$

Choose any Hermite-Biehler function $E$ generating $\mathcal{B}_{r}=\left(\mathrm{PW}_{s}, \mu\right)$ and define the functions $P: z \mapsto e^{i s z} E^{\sharp}, P^{*}: z \mapsto e^{i s z} E$. Using formula (20), it is not difficult to see that

$$
k_{2 s, \lambda}: z \mapsto-\frac{1}{2 \pi i} \frac{P^{*}(z) \overline{P^{*}(\lambda)}-P(z) \overline{P(\lambda)}}{z-\bar{\lambda}}, \quad z \in \mathbb{C},
$$

is the reproducing kernel of $\left(\mathrm{PW}_{[0,2 s]}, \mu\right)$ at $\lambda \in \mathbb{C}$. By construction, we have $\mu=w d x+\mu_{\mathbf{s}}$, hence $\|f\|_{H^{2}(w)} \leqslant\|f\|_{L^{2}(\mu)}$ for every $f \in\left(\mathrm{PW}_{[0,2 s]}, \mu\right)$. It follows that $\left\|k_{2 s, \lambda}\right\|_{L^{2}(\mu)}$ does not exceed the norm of the reproducing kernel of the space $H^{2}(w)$ at $\lambda \in \mathbb{C}^{+}$. Since the reproducing kernel of $H^{2}(w)$ is given by

$$
k_{H^{2}(w), \lambda}: z \mapsto-\frac{1}{2 \pi i} \frac{D_{\mu}^{-1}(z) \overline{D_{\mu}^{-1}(\lambda)}}{z-\bar{\lambda}},
$$

this yields the useful inequality $\left|P^{*}(\lambda)\right|^{2}-|P(\lambda)|^{2} \leqslant\left|D_{\mu}(\lambda)\right|^{-2}$ for every $\lambda \in \mathbb{C}^{+}$.

2.4. Reduction of Dirac systems to canonical Hamiltonian systems. It is well-known that Dirac systems could be rewritten as canonical Hamiltonian systems, see, e.g., Section 3 in [12]. For the reader convenience, we reproduce this observation. Consider the Dirac system

$$
J N^{\prime}(t, z)+Q(t) N(t, z)=z N(t, z), \quad t \geqslant 0, \quad N(0, z)=\left(\begin{array}{cc}
1 & 0 \\
0 & 1
\end{array}\right),
$$

and let $N_{0}(t)=N(0, t), t \geqslant 0$. Define $M=M(t, z)$ by $N(t, z)=N_{0}(t) M(t, z)$. Then we have

$$
\begin{aligned}
z N_{0}(t) M(t, z) & =J N_{0}^{\prime}(t) M(t, z)+J N_{0}(t) J^{*} J M^{\prime}(t, z)+Q N_{0}(t) M(t, z) \\
& =J N_{0}(t) J^{*} J M^{\prime}(t, z)=\left(N_{0}^{*}(t)\right)^{-1} J M^{\prime}(t, z),
\end{aligned}
$$

because $J A J^{*}=\left(A^{*}\right)^{-1}$ for every real matrix $A$ with unit determinant. It follows that $M$ solves the Cauchy problem (10) for the Hamiltonian $\mathcal{H}=N_{0}^{*} N_{0}$ on $\mathbb{R}_{+}$. In particular, for the potential $Q=\left(\begin{array}{cc}0 & q \\ q & 0\end{array}\right)$ on $\mathbb{R}_{+}$we have

$$
N_{0}(t)=\left(\begin{array}{cc}
e^{-g(t)} & 0 \\
0 & e^{g(t)}
\end{array}\right), \quad \mathcal{H}(t)=\left(\begin{array}{cc}
e^{-2 g(t)} & 0 \\
0 & e^{2 g(t)}
\end{array}\right), \quad t \geqslant 0,
$$

where $g(t)=\int_{0}^{t} q(s) d s$. Similarly, for the potential $Q=\left(\begin{array}{cc}q & 0 \\ 0 & -q\end{array}\right)$ on $\mathbb{R}_{+}$we have

$$
N_{0}(t)=\left(\begin{array}{cc}
\cosh g(t) & \sinh g(t) \\
\sinh g(t) & \cosh g(t)
\end{array}\right), \quad \mathcal{H}(t)=\left(\begin{array}{cc}
\cosh 2 g(t) & \sinh 2 g(t) \\
\sinh 2 g(t) & \cosh 2 g(t)
\end{array}\right), \quad t \geqslant 0 .
$$

Next, let us show that the spectral measures of $\mathcal{H}$ and $\mathcal{D}_{Q}$ coincide. Let $\Psi$ be the solution of (5), and let $\Theta$ be defined by (11). From the relation $N=N_{0} M$ 
we see that $\Psi(t, z)=N_{0}(t) \Theta(t, z)$ for all $t \geqslant 0$ and all $z \in \mathbb{C}$. Using the identity $\mathcal{H}=N_{0}^{*} N_{0}$, we get

$$
\int_{0}^{\infty}\langle\mathcal{H}(t) X(t), \Theta(t, \bar{z})\rangle_{\mathbb{C}^{2}}=\int_{0}^{\infty}\left\langle N_{0}(t) X(t), \Psi(t, \bar{z})\right\rangle_{\mathbb{C}^{2}},
$$

for every function $X \in L^{2}\left(\mathbb{R}_{+}, \mathbb{C}^{2}\right)$ with compact support. Moreover, the mapping $X \mapsto N_{0} X$ is the unitary operator from $L^{2}(\mathcal{H})$ to $L^{2}\left(\mathbb{R}_{+}, \mathbb{C}^{2}\right)$. It follows that operators $\mathcal{F}_{Q}: L^{2}\left(\mathbb{R}_{+}, \mathbb{C}^{2}\right) \rightarrow L^{2}(\mu), \mathcal{W}_{\mathcal{H}}: L^{2}\left(\mathbb{R}_{+}, \mathbb{C}^{2}\right) \rightarrow L^{2}(\mu)$ are isometric or not simultaneously. In other words, the spectral measures of $\mathcal{H}$ and $\mathcal{D}_{Q}$ coincide.

\section{KhrushcheV formula AND PRoOf of Theorem 2}

Let $\mathcal{H}$ be a singular nontrivial Hamiltonian, and let $\left\{m_{r}\right\}_{r} \geqslant 0$ be the family of Weyl functions constructed in Section 2, Recall that $m_{r}(i)=i \mathcal{I}_{\mathcal{H}}(r)+\mathcal{R}_{\mathcal{H}}(r)$ and $\mathcal{I}_{\mathcal{H}}(r)>0$ for every $r>0$. Define the Schur family $\left\{f_{r}\right\}_{r \geqslant 0}$ associated to $\mathcal{H}$ by

$$
m_{r}(z)=i \mathcal{I}_{\mathcal{H}}(r) \frac{1+B_{i}(z) f_{r}(z)}{1-B_{i}(z) f_{r}(z)}+\mathcal{R}_{\mathcal{H}}(r), \quad z \in \mathbb{C}^{+}, \quad r \geqslant 0
$$

where $B_{i}: z \mapsto \frac{z-i}{z+i}$ is the Blaschke factor in $\mathbb{C}^{+}$. By construction, the functions $f_{r}$ are analytic on $\mathbb{C}^{+}$. We also have

$$
\operatorname{Im} m_{r}(z)=\mathcal{I}_{\mathcal{H}}(r) \operatorname{Re}\left(\frac{1+B_{i}(z) f_{r}(z)}{1-B_{i}(z) f_{r}(z)}\right)=\mathcal{I}_{\mathcal{H}}(r) \frac{1-\left|B_{i}(z) f_{r}(z)\right|^{2}}{\left|1-B_{i}(z) f_{r}(z)\right|^{2}} .
$$

Since $\mathcal{I}_{\mathcal{H}}(r)>0$ for every $r \geqslant 0$, we see that $\left|B_{i}(z) f_{r}(z)\right|<1$ for $z \in \mathbb{C}^{+}$. By Schwarz lemma, this implies $\left|f_{r}(z)\right| \leqslant 1$ for $z \in \mathbb{C}^{+}$. Thus, the family $\left\{f_{r}\right\}_{r \geqslant 0}$ consists of contracting analytic functions on $\mathbb{C}^{+}$. Let us represent the Möbius transform $\tau: w \mapsto \mathcal{I}_{\mathcal{H}}(r) w+\mathcal{R}_{\mathcal{H}}(r)$ in the form

$$
\tau: w \mapsto \frac{\sqrt{\mathcal{I}_{\mathcal{H}}(r)} w+\mathcal{R}_{\mathcal{H}}(r) / \sqrt{\mathcal{I}_{\mathcal{H}}(r)}}{0 \cdot w+1 / \sqrt{\mathcal{I}_{\mathcal{H}}(r)}} .
$$

Let $\Theta^{ \pm}, \Phi^{ \pm}$be the entries of the solution $M$ of Cauchy problem (10), see (11). For $r>0$, define the entire functions $\tilde{\Theta}_{r}^{ \pm}, \tilde{\Phi}_{r}^{ \pm}$by

$$
\left(\begin{array}{cc}
\tilde{\Phi}_{r}^{-} & \tilde{\Phi}_{r}^{+} \\
\tilde{\Theta}_{r}^{-} & \tilde{\Theta}_{r}^{+}
\end{array}\right)=\left(\begin{array}{cc}
\Phi^{-}(r, \cdot) & \Phi^{+}(r, \cdot) \\
\Theta^{-}(r, \cdot) & \Theta^{+}(r, \cdot)
\end{array}\right)\left(\begin{array}{cc}
\sqrt{\mathcal{I}_{\mathcal{H}}(r)} & \mathcal{R}_{\mathcal{H}}(r) / \sqrt{\mathcal{I}_{\mathcal{H}}(r)} \\
0 & 1 / \sqrt{\mathcal{I}_{\mathcal{H}}(r)}
\end{array}\right) .
$$

Using (23) and (24), we see that

$$
\frac{\tilde{\Phi}^{-}(r, z) w+\tilde{\Phi}^{+}(r, z)}{\tilde{\Theta}^{-}(r, z) w+\tilde{\Theta}^{+}(r, z)}=\frac{\Phi^{-}(r, z) \tau(w)+\Phi^{+}(r, z)}{\Theta^{-}(r, z) \tau(w)+\Theta^{+}(r, z)}, \quad w \in \mathbb{C} .
$$

This formula will be used in the proof of Lemma 5 below. Relation (24) can be rewritten in the form

$$
\left(\begin{array}{cc}
\tilde{\Theta}_{r}^{+} & \tilde{\Phi}_{r}^{+} \\
\tilde{\Theta}_{r}^{-} & \tilde{\Phi}_{r}^{-}
\end{array}\right)=\left(\begin{array}{cc}
1 / \sqrt{\mathcal{I}_{\mathcal{H}}(r)} & \mathcal{R}_{\mathcal{H}}(r) / \sqrt{\mathcal{I}_{\mathcal{H}}(r)} \\
0 & \sqrt{\mathcal{I}_{\mathcal{H}}(r)}
\end{array}\right)\left(\begin{array}{cc}
\Theta^{+}(r, \cdot) & \Phi^{+}(r, \cdot) \\
\Theta^{-}(r, \cdot) & \Phi^{-}(r, \cdot)
\end{array}\right) .
$$

The functions $\tilde{\Theta}_{r}^{ \pm}, \tilde{\Phi}_{r}^{ \pm}$take real values on the real line and

$$
\tilde{\Theta}_{r}^{+}(z) \tilde{\Phi}_{r}^{-}(z)-\tilde{\Theta}_{r}^{-}(z) \tilde{\Phi}_{r}^{+}(z)=1, \quad z \in \mathbb{C},
$$


as can be easily seen from (26) by calculating determinants and using the fact that $\operatorname{det} M(r, z)=\operatorname{det} M(0, z)=1$. Next, define the entire functions

$$
\begin{array}{lll}
\tilde{E}_{r}: z \mapsto \tilde{\Theta}_{r}^{+}(z)+i \tilde{\Theta}_{r}^{-}(z), & & \tilde{E}_{r}^{\sharp}: z \mapsto \tilde{\Theta}_{r}^{+}(z)-i \tilde{\Theta}_{r}^{-}(z) \\
\tilde{F}_{r}: z \mapsto \tilde{\Phi}_{r}^{+}(z)+i \tilde{\Phi}_{r}^{-}(z), & & \tilde{F}_{r}^{\sharp}: z \mapsto \tilde{\Phi}_{r}^{+}(z)-i \tilde{\Phi}_{r}^{-}(z) .
\end{array}
$$

Since the functions $\Theta_{r}^{ \pm}$take real values on the real line, we have $\left|\tilde{E}_{r}(x)\right|=\left|\tilde{E}_{r}^{\sharp}(x)\right|$ for $x \in \mathbb{R}$. The same is true for the functions $\tilde{F}_{r}, \tilde{F}_{r}^{\sharp}$.

Lemma 4. The measure $\left|\tilde{E}_{r}(x)\right|^{-2} d x$ is the spectral measure for $\widehat{\mathcal{H}}_{r}$. The function $\tilde{E}_{r}$ belongs to the Hermite-Biehler class and generates the subspace $\mathcal{B}_{r}=\mathcal{W}_{\mathcal{H}} H_{r}$ of de Branges chain associated to $\mathcal{H}$.

Proof. Let us apply assertion (b) of Lemma 1 to the Hamiltonian $\widehat{\mathcal{H}}_{r}$. By definition, the Hamiltonian $\left(\widehat{\mathcal{H}}_{r}\right)_{r}$ coincides with the constant matrix

$$
\left(\widehat{\mathcal{H}}_{r}\right)_{r}=\left(\begin{array}{cc}
1 / \mathcal{I}_{\mathcal{H}}(r) & \mathcal{R}_{\mathcal{H}}(r) / \mathcal{I}_{\mathcal{H}}(r) \\
\mathcal{R}_{\mathcal{H}}(r) / \mathcal{I}_{\mathcal{H}}(r) & \left(\mathcal{I}_{\mathcal{H}}^{2}(r)+\mathcal{R}_{\mathcal{H}}^{2}(r)\right) / \mathcal{I}_{\mathcal{H}}(r)
\end{array}\right)
$$

The Weyl function of this Hamiltonian equals

$$
m_{\left(\widehat{\mathcal{H}}_{r}\right)_{r}}=i \mathcal{I}_{\mathcal{H}}(r)+\mathcal{R}_{\mathcal{H}}(r),
$$

hence the spectral measure of $\left(\widehat{\mathcal{H}}_{r}\right)_{r}$ is $\mathcal{I}_{\mathcal{H}}(r) d x$. Since $\widehat{\mathcal{H}}_{r}=\mathcal{H}$ on $[0, r]$, we have $\hat{F}_{r}(z)=\Theta^{+}(r, z)+m_{\left(\widehat{\mathcal{H}}_{r}\right)_{r}}(z) \Theta^{-}(r, z)$ for the function $\hat{F}_{r}$ from Lemma 1 for $\widehat{\mathcal{H}}_{r}$. Then assertion $(b)$ of Lemma 1 says that for almost all $x \in \mathbb{R}$ we have

$$
\hat{w}_{r}(x)=\frac{\mathcal{I}_{\mathcal{H}}(r)}{\left|\Theta^{+}(r, x)+\left(i \mathcal{I}_{\mathcal{H}}(r)+\mathcal{R}_{\mathcal{H}}(r)\right) \Theta^{-}(r, x)\right|^{2}}=\frac{1}{\left|\tilde{E}_{r}(x)\right|^{2}},
$$

where $\hat{w}_{r}$ is the density of the absolutely continuous part of the spectral measure $\hat{\mu}_{r}$ of $\widehat{\mathcal{H}}_{r}$. Note that for every $x \in \mathbb{R}$ we have $\lim _{\varepsilon \rightarrow 0}\left|F_{r}(x+i \varepsilon)\right|>0$. Indeed, this follows from the fact that $\Theta^{ \pm}(r, \cdot)$ are real analytic functions whose zero sets do not intersect: $\operatorname{det} M(r, z)=1$. From assertion $(a)$ of Lemma 1 we see that $\lim _{\varepsilon \rightarrow 0} \operatorname{Im} \hat{m}_{r}(x+i \varepsilon)$ exists and finite for every $x \in \mathbb{R}$, hence the singular part of $\hat{\mu}_{r}$ is zero. Formula (29) now says that $\left|\tilde{E}_{r}(x)\right|^{-2} d x$ is the spectral measure for $\widehat{\mathcal{H}}_{r}$. A lengthy but elementary calculation shows that

$$
\left|\tilde{E}_{r}(z)\right|^{2}-\left|\tilde{E}_{r}^{\sharp}(z)\right|^{2}=4 \operatorname{Im}\left(\Theta^{+}(z) \overline{\Theta^{-}(z)}\right)=\left|E_{r}(z)\right|^{2}-\left|E_{r}^{\sharp}(z)\right|^{2}, \quad z \in \mathbb{C},
$$

where $E_{r}$ is defined by (19). Since $E_{r}$ belongs to the Hermite-Biehler class, the above formula shows that the same is true for the function $\tilde{E}_{r}$. Moreover, this formula implies that

$$
\tilde{E}_{r}(z) \overline{\tilde{E}_{r}(\lambda)}-\tilde{E}_{r}^{\sharp}(z) \overline{\tilde{E}_{r}^{\sharp}(\lambda)}=E_{r}(z) \overline{E_{r}(\lambda)}-E_{r}^{\sharp}(z) \overline{E_{r}^{\sharp}(\lambda)}, \quad z, \lambda \in \mathbb{C} .
$$

Hence the reproducing kernels generated by $E_{r}$ and $\tilde{E}_{r}$ coincide, see (20). It follows that $\tilde{E}_{r}$ generates the subspace $\mathcal{B}_{r}$.

Next lemma is the analogue of Theorem 2 (page 173) in Khrushchev paper [8].

Lemma 5. For every $r \geqslant 0$ and for almost all $x \in \mathbb{R}$ we have

$$
\left|\tilde{E}_{r}(x)\right|^{2} w(x)=\frac{1-\left|\tilde{f}_{r}(x)\right|^{2}}{\left|1-\theta_{r}(x) \tilde{f}_{r}(x)\right|^{2}},
$$

where $\tilde{f}_{r}=B_{i} f_{r}$, and $\theta_{r}: z \mapsto \frac{\tilde{E}_{r}^{*}(z)}{\tilde{E}_{r}(z)}$ is the inner function in $\mathbb{C}^{+}$. 
Proof. Assertion (a) of Lemma 1 says

$$
m(z)=\frac{\Phi^{+}(r, z)+m_{r}(z) \Phi^{-}(r, z)}{\Theta^{+}(r, z)+m_{r}(z) \Theta^{-}(r, z)}, \quad z \in \mathbb{C}^{+}, \quad r \geqslant 0 .
$$

From (22) and (25) we get

$$
m=\frac{\tilde{\Phi}_{r}^{+}+i \tilde{\Phi}_{r}^{-} \frac{1+\tilde{f}_{r}}{1-\tilde{f}_{r}}}{\tilde{\Theta}_{r}^{+}+i \tilde{\Theta}_{r}^{-} \frac{1+\tilde{f}_{r}}{1-\tilde{f}_{r}}}=\frac{\left(\tilde{\Phi}_{r}^{+}+i \tilde{\Phi}_{r}^{-}\right)+\left(-\tilde{\Phi}_{r}^{+}+i \tilde{\Phi}_{r}^{-}\right) \tilde{f}_{r}}{\left(\tilde{\Theta}_{r}^{+}+i \tilde{\Theta}_{r}^{-}\right)+\left(-\tilde{\Theta}_{r}^{+}+i \tilde{\Theta}_{r}^{-}\right) \tilde{f}_{r}}=\frac{\tilde{F}_{r}-\tilde{F}_{r}^{\sharp} \tilde{f}_{r}}{\tilde{E}_{r}-\tilde{E}_{r}^{\sharp} \tilde{f}_{r}}
$$

in the upper half-plane $\mathbb{C}^{+}$. Taking into account (27) and the fact that $\tilde{\Theta}^{ \pm}, \tilde{\Phi}^{ \pm}$ are real on $\mathbb{R}$, we conclude that $\operatorname{Im} \tilde{F}_{r} \tilde{E}_{r}=\operatorname{Im}\left(\tilde{\Phi}_{r}^{+}+i \tilde{\Phi}_{r}^{-}\right)\left(\tilde{\Theta}_{r}^{+}-i \tilde{\Theta}_{r}^{-}\right)=1$ on the real line $\mathbb{R}$. Analogously, $\operatorname{Im} \tilde{F}_{r}^{\sharp}(x) \overline{\tilde{E}_{r}^{\sharp}(x)}=-1$ on $\mathbb{R}$. Next, the bounded analytic function $\tilde{f}_{r}$ has a non-tangential limit at almost every point $x \in \mathbb{R}$, and, moreover,

$$
\operatorname{Im}\left(\tilde{F}_{r}^{\sharp}(x) \overline{\tilde{E}_{r}(x)} \tilde{f}_{r}(x)+\tilde{F}_{r}(x) \overline{\tilde{E}_{r}^{\sharp}(x) \tilde{f}_{r}(x)}\right)=0,
$$

because $\tilde{E}_{r}^{\sharp}=\overline{\tilde{E}_{r}}, \tilde{F}_{r}^{\sharp}={\overline{F_{r}}}_{r}$ on $\mathbb{R}$. It follows that for almost all $x \in \mathbb{R}$ we have

$$
\operatorname{Im} m(x)=\operatorname{Im} \frac{\tilde{F}_{r}(x) \overline{\tilde{E}_{r}(x)}+\tilde{F}_{r}^{\sharp}(x) \overline{\tilde{E}_{r}^{\sharp}(x)}\left|\tilde{f}_{r}(x)\right|^{2}}{\left|\tilde{E}_{r}(x)-\tilde{E}_{r}^{\sharp}(x) \tilde{f}_{r}(x)\right|^{2}}=\frac{1-\left|\tilde{f}_{r}(x)\right|^{2}}{\left|\tilde{E}_{r}(x)-\tilde{E}_{r}^{\sharp}(x) \tilde{f}_{r}(x)\right|^{2}} .
$$

It remains to note that $w(x)=\operatorname{Im} m(x)$ almost everywhere on $\mathbb{R}$, hence

$$
\left|\tilde{E}_{r}(x)\right|^{2} w(x)=\frac{\left|\tilde{E}_{r}(x)\right|^{2}\left(1-\left|\tilde{f}_{r}(x)\right|^{2}\right)}{\left|\tilde{E}_{r}(z)-\tilde{E}_{r}^{\sharp}(z) \tilde{f}_{r}(z)\right|^{2}}=\frac{1-\left|\tilde{f}_{r}(x)\right|^{2}}{\left|1-\theta_{r}(x) \tilde{f}_{r}(x)\right|^{2}},
$$

as required. Since $\tilde{E}_{r}$ belongs to the Hermite-Biehler class, the function $\theta_{r}$ is inner in $\mathbb{C}^{+}$.

We are ready to prove Theorem 2, It is interesting to note that the proof below is very close to the proof of Theorem 2.5 in Khrushchev paper [8]. It seems that some other results from [8] related to Schur functions also have analogues for canonical Hamiltonian systems.

Proof of Theorem 2, For $r>0$, define the function $\tilde{E}_{r}$ by (28). By Lemma 4 , $\tilde{E}_{r}$ belongs to the Hermite-Biehler class and generates the subspace $\mathcal{B}_{r}=\mathcal{W}_{\mathcal{H}} H_{r}$. Moreover, $\left|\tilde{E}_{r}(x)\right|^{-2} d x$ is the spectral measure for the Hamiltonian $\widehat{\mathcal{H}}_{r}$. Let $\hat{m}_{r}$ denote the Weyl function (13) of $\widehat{\mathcal{H}}_{r}$. Since $\widehat{\mathcal{H}}_{r}$ coincides with $\mathcal{H}$ on $[0, r)$, the standard argument based on nesting circle analysis gives $\lim _{r \rightarrow \infty} \operatorname{Im} \hat{m}_{r}(i)=\operatorname{Im} m(i)$, see Lemma 4.1 in [1] for more details. The last relation can be rewritten in the form

$$
\lim _{r \rightarrow \infty} \int_{\mathbb{R}} \frac{1}{\left|\tilde{E}_{r}(x)\right|^{2}} d P(x)=\int_{\mathbb{R}} w(x) d P(x),
$$

where we denoted $d P(x)=\frac{1}{\pi} \frac{d x}{x^{2}+1}$. Next, by Lemma 2 we have

$$
\lim _{r \rightarrow \infty} \mathcal{K}_{\widehat{\mathcal{H}}_{r}}(0)=\mathcal{K}_{\mathcal{H}}(0) .
$$

Using this fact, the definition of $\mathcal{K}_{\mathcal{H}}$, and relation (31), we see that

$$
\lim _{r \rightarrow \infty} \int_{\mathbb{R}} \log \frac{1}{\left|\tilde{E}_{r}(x)\right|^{2}} d P(x)=\int_{\mathbb{R}} \log w(x) d P(x) .
$$


By Lemma 5, we have

$$
\int_{\mathbb{R}} \log \left(\left|\tilde{E}_{r}\right|^{2} w\right) d P=\int_{\mathbb{R}} \log \frac{1-\left|\tilde{f}_{r}\right|^{2}}{\left|1-\theta_{r} \tilde{f}_{r}\right|^{2}} d P=\int_{\mathbb{R}} \log \left(1-\left|\tilde{f}_{r}\right|^{2}\right) d P,
$$

where we used the fact that $\int_{\mathbb{R}} \log \left|1-\theta_{r} \tilde{f}_{r}\right| d P=\log \left|1-\theta_{r}(i) \tilde{f}_{r}(i)\right|=0$ by the mean value theorem for harmonic functions. Relations (32), (33) now give us

$$
\lim _{r \rightarrow \infty} \int_{\mathbb{R}} \log \left(1-\left|\tilde{f}_{r}\right|^{2}\right) d P=0 .
$$

Denote $\log ^{+} x=\max (\log x, 0)$. We have $\int_{\mathbb{R}}|\log g| d P \leqslant 2 \int_{\mathbb{R}} \log ^{+} g d P$ for every function $g \in L^{1}(P)$ with $\int_{\mathbb{R}} \log g d P=0$. Hence (34) implies

$$
\limsup _{r \rightarrow \infty} \int_{\mathbb{R}}\left|\log \frac{1}{\left|\tilde{E}_{r}\right|^{2}}-\log w\right| d P \leqslant 2 \limsup _{r \rightarrow \infty} \int_{\mathbb{R}} \log ^{+} \frac{1}{\left|\tilde{E}_{r}\right|^{2} w} d P .
$$

Applying Lemma 5 once more, we obtain

$$
\begin{aligned}
\int_{\mathbb{R}} \log ^{+} \frac{1}{\left|\tilde{E}_{r}\right|^{2} w} d P & =\int_{\mathbb{R}} \log ^{+} \frac{\left|1-\theta_{r} \tilde{f}_{r}\right|^{2}}{1-\left|\tilde{f}_{r}\right|^{2}} d P \leqslant \int_{\mathbb{R}} \log \frac{\left(1+\left|\tilde{f}_{r}\right|\right)^{2}}{1-\left|\tilde{f}_{r}\right|^{2}} d P \\
& \leqslant \int_{\mathbb{R}} \log \frac{1}{1-\left|\tilde{f}_{r}\right|^{2}} d P+2 \int_{\mathbb{R}}\left|\tilde{f}_{r}\right| d P .
\end{aligned}
$$

Using the fact that $x \leqslant \log \frac{1}{1-x}$ for $0 \leqslant x<1$ and $P(\mathbb{R})=1$, we can estimate

$$
\left(\int_{\mathbb{R}}\left|\tilde{f}_{r}\right| d P\right)^{2} \leqslant \int_{\mathbb{R}}\left|\tilde{f}_{r}\right|^{2} d P \leqslant \int_{\mathbb{R}} \log \frac{1}{1-\left|\tilde{f}_{r}\right|^{2}} d P
$$

which tends to zero by (34). We now see that (35) implies

$$
\lim _{r \rightarrow \infty} \int_{\mathbb{R}}\left|\log \frac{1}{\left|\tilde{E}_{r}(x)\right|^{2}}-\log w(x)\right| d P(x)=0 .
$$

This completes the proof of the theorem.

\section{Regularized Krein orthogonal entire functions}

Consider a Hamiltonian $\mathcal{H}=\left(\begin{array}{cc}h_{1} & h \\ h & h_{2}\end{array}\right)$ on $\mathbb{R}_{+}$such that $\operatorname{det} \mathcal{H}(t)=1$ for almost all $t \in \mathbb{R}_{+}$. Let $\mu$ be the spectral measure of $\mathcal{H}$. Assume that $\mu=w d x+\mu_{\mathbf{s}}$ belongs to the Szegó class $\mathrm{Sz}(\mathbb{R})$. To simplify notations, we set $I=\mathcal{I}_{\mathcal{H}}, R=\mathcal{R}_{\mathcal{H}}, K=\mathcal{K}_{\mathcal{H}}$ for the rest of this section. For $r>0$, define entire functions $\tilde{P}_{r}, \tilde{P}_{r}^{*}$ by

$$
\tilde{P}_{2 r}: z \mapsto e^{i r z-i u(r)} \tilde{E}_{r}^{\sharp}(z), \quad \tilde{P}_{2 r}^{*}: z \mapsto e^{i r z+i u(r)} \tilde{E}_{r}(z), \quad z \in \mathbb{C},
$$

where $u: r \mapsto \int_{0}^{r} \frac{R^{\prime}(t)}{2 I(t)} d t$ and $\tilde{E}_{r}, \tilde{E}_{r}^{\sharp}$ are given by (28). Since $R, I$ are locally absolutely continuous and $I$ is strictly positive, the function $u$ is correctly defined on $\mathbb{R}_{+}$and is locally absolutely continuous. Functions $\tilde{P}_{r}, \tilde{P}_{r}^{*}$ could be regarded as regularized versions of Krein's orthogonal entire functions $P_{r}, P_{r}^{*}$ introduced by Krein in [9], see [5] for their modern theory.

Lemma 6. For every $r>0$ the function $\tilde{P}_{2 r}^{*}$ is outer in $\mathbb{C}^{+}$. 
Proof. Fix $r>0$. The function $\tilde{E}_{r}$ belongs the Hermite-Biehler class and

$$
\limsup _{|z| \rightarrow \infty} \frac{\log \left|\tilde{E}_{r}(z)\right|}{|z|}=\limsup _{y \rightarrow+\infty} \frac{\log \left|\tilde{E}_{r}(i y)\right|}{y}=r,
$$

see Section 6 in [12]. It follows that either $\tilde{E}_{r}=e^{-i r z}$ or there exists a point $\lambda \in \mathbb{C}$, $\operatorname{Im} \lambda<0$, such that $\tilde{E}_{r}(\lambda)=0$. In the first case we have $\tilde{P}_{2 r}^{*}=1$ and there is nothing to prove. In the second case consider the reproducing kernel $k_{2 r, \bar{\lambda}}$ of the space $\left(\mathrm{PW}_{[0,2 r]}, \mu\right)$. Using formula (21), we see that

$$
k_{2 r, \bar{\lambda}}(z)=-\frac{1}{2 \pi i} \frac{\tilde{P}_{2 r}^{*}(z) \overline{\tilde{P}_{2 r}^{*}(\bar{\lambda})}}{z-\lambda}, \quad z \in \mathbb{C},
$$

belongs to the weighted Hardy space $H^{2}(w)$. Hence $k_{2 r, \lambda} / D_{\mu} \in H^{2}\left(\mathbb{C}^{+}\right)$and we can use Smirnov-Nevanlinna factorization for functions of bounded type in $\mathbb{C}^{+}$(see, e.g., Theorem 9 in [3]) to find an inner function $\theta$ and an outer function $h$ such that $\tilde{P}_{2 r}^{*}=\theta h$ in $\mathbb{C}^{+}$. Since $\tilde{P}_{2 r}^{*}$ does not vanish on $\{z \in \mathbb{C}: \operatorname{Im} z \geqslant 0\}$, the function $\theta$ has the form $\theta=e^{i s z}$ for some $s \geqslant 0$. Moreover, we have

$$
\limsup _{y \rightarrow+\infty} \frac{\log \left|\tilde{E}_{r}(i y)\right|}{y}=\limsup _{y \rightarrow+\infty} \frac{\log \left|e^{r y} \tilde{P}_{2 r}^{*}(i y)\right|}{y}=r+\limsup _{y \rightarrow+\infty} \frac{\log |\theta(i y)|}{y}=r-s,
$$

because $\log |h(i y)|=o(y)$ is the Poisson extension of a function from $L^{1}(P)$. Comparing this formula with (38), we see that $s=0$. Hence, the function $\tilde{P}_{r}^{*}$ is outer in $\mathbb{C}^{+}$, as required.

Lemma 7. The functions $I^{\prime} / I, R^{\prime} / I,\left(R^{\prime} / I\right)^{2}\left(I h_{1}\right)^{-1}, 1-I h_{1}, 1-1 / I h_{1}$ belong to the space $L^{1}\left(\mathbb{R}_{+}\right)+L^{2}\left(\mathbb{R}_{+}\right)$. We also have $K^{\prime} \in L^{1}\left(\mathbb{R}_{+}\right)$.

Proof. The fact that that $K^{\prime} \in L^{1}(\mathbb{R})$ is the direct consequence of Lemma 2 , Relations in Lemma 3 can be rewritten in the form

$$
\begin{aligned}
-K^{\prime} & =\left(I h_{1}+\frac{1}{I h_{1}}-2\right)+\frac{1}{4}\left(\frac{R^{\prime}}{I}\right)^{2} \frac{1}{I h_{1}}, \\
\frac{I^{\prime}}{I} & =\left(I h_{1}-\frac{1}{I h_{1}}\right)-\frac{1}{4}\left(\frac{R^{\prime}}{I}\right)^{2} \frac{1}{I h_{1}}, \\
\frac{R^{\prime}}{I} & =2 R h_{1}-2 h,
\end{aligned}
$$

almost everywhere on $\mathbb{R}_{+}$. Since $K^{\prime} \in L^{1}\left(\mathbb{R}_{+}\right)$, and the functions

$$
g=I h_{1}+\frac{1}{I h_{1}}-2, \quad\left(\frac{R^{\prime}}{I}\right)^{2} \frac{1}{I h_{1}},
$$

are nonnegative, they belong to $L^{1}\left(\mathbb{R}_{+}\right)$. Denote $S_{1}=\left\{t \in \mathbb{R}_{+}: 1 / 2 \leqslant I h_{1} \leqslant 2\right\}$. The function $g$ is comparable to $I h_{1}+\frac{1}{I h_{1}}$ on $\mathbb{R}_{+} \backslash S_{1}$, while on $S_{1}$ we have

$$
c_{1}\left|1-\frac{1}{I h_{1}}\right|^{2}+c_{1}\left|1-I h_{1}\right|^{2} \leqslant g \leqslant c_{2}\left|1-\frac{1}{I h_{1}}\right|^{2}+c_{2}\left|1-I h_{1}\right|^{2}
$$

for some positive constants $c_{1}, c_{2}$. Hence $1-I h_{1}, 1-1 / I h_{1}$ are in $L^{1}\left(\mathbb{R}_{+}\right)+L^{2}\left(\mathbb{R}_{+}\right)$. From (39), (40) we also see that $I^{\prime} / I \in L^{1}\left(\mathbb{R}_{+}\right)+L^{2}\left(\mathbb{R}_{+}\right)$. Formula (39) implies 
that $R^{\prime} / I \in L^{2}\left(S_{1}\right)$. Moreover, on $S_{2}=\mathbb{R}_{+} \backslash S_{1}$ we have

$$
\left(\int_{S_{2}}\left|\frac{R^{\prime}(t)}{I(t)}\right| d t\right)^{2} \leqslant \int_{S_{2}}\left(\frac{R^{\prime}(t)}{I(t)}\right)^{2} \frac{1}{I h_{1}(t)} d t \cdot \int_{S_{2}} I h_{1}(t) d t<+\infty
$$

where we used the fact that $g \in L^{1}\left(\mathbb{R}_{+}\right)$is comparable to $I h_{1}+\frac{1}{I h_{1}}$ on $S_{2}$. Thus, we have $R^{\prime} / I \in L^{1}\left(\mathbb{R}_{+}\right)+L^{2}\left(\mathbb{R}_{+}\right)$and lemma follows.

Next lemma concerns an analogue of the relation $\frac{\partial}{\partial r} P_{r}^{*}(z)=-A(r) P_{r}(z)$ (see Theorem 4.9 in [5]) for functions $\tilde{P}_{r}, \tilde{P}_{r}^{*}$.

Lemma 8. For every $z \in \mathbb{C}$, the function $r \mapsto \tilde{P}_{r}^{*}(z)$ is locally absolutely continuous on $\mathbb{R}_{+}$. Moreover, we have

$$
\frac{\partial}{\partial r} \tilde{P}_{2 r}^{*}(z)=-\frac{1}{2}(z-i) e^{2 i u(r)}\left(\frac{R^{\prime}(r)}{I(r)}+i \frac{I^{\prime}(r)}{I(r)}\right) \tilde{P}_{2 r}(z)+\frac{i}{2} z K^{\prime}(r) \tilde{P}_{2 r}^{*}(z),
$$

for almost all $r>0$.

Proof. Relation (26) can we written in the form $\tilde{\Theta}=G \Theta$, where the matrixfunction $G$ is given by

$$
G(r)=\left(\begin{array}{cc}
1 / \sqrt{I(r)} & R(r) / \sqrt{I(r)} \\
0 & \sqrt{I(r)}
\end{array}\right), \quad r \geqslant 0 .
$$

Differentiating, we get

$$
J \tilde{\Theta}^{\prime}=J G^{\prime} \Theta+z J G J^{*} \mathcal{H} \Theta=J G^{\prime} G^{-1} \tilde{\Theta}+z J G J^{*} \mathcal{H} G^{-1} \tilde{\Theta} .
$$

For the matrix $G$ we have

$$
\begin{aligned}
G^{\prime} & =\frac{1}{2} \frac{I^{\prime}}{I}\left(\begin{array}{cc}
-1 / \sqrt{I} & -R / \sqrt{I} \\
0 & \sqrt{I}
\end{array}\right)+\left(\begin{array}{cc}
0 & R^{\prime} / \sqrt{I} \\
0 & 0
\end{array}\right), \\
G^{-1} & =\left(\begin{array}{cc}
\sqrt{I} & -R / \sqrt{I} \\
0 & 1 / \sqrt{I}
\end{array}\right)=\left(J G J^{*}\right)^{*}, \\
J G^{\prime} G^{-1} & =-\frac{1}{2} \frac{I^{\prime}}{I}\left(\begin{array}{cc}
0 & 1 \\
1 & 0
\end{array}\right)+\left(\begin{array}{cc}
0 & 0 \\
0 & R^{\prime} / I
\end{array}\right), \\
J G J^{*} \mathcal{H} G^{-1} & =\left(\begin{array}{cc}
I h_{1} & -R h_{1}+h \\
-R h_{1}+h & \left(R^{2} h_{1}-2 R h+h_{2}\right) / I
\end{array}\right) .
\end{aligned}
$$

Substituting these expressions into the formula for $J \tilde{\Theta}^{\prime}$ and using identity $R^{\prime} / I=$ $2 R h_{1}-2 h$ from Lemma 3 we obtain

$$
\begin{aligned}
& \frac{\partial}{\partial r} \tilde{\Theta}^{-}=\frac{1}{2}\left(I^{\prime} / I+z R^{\prime} / I\right) \tilde{\Theta}^{-}-z I h_{1} \tilde{\Theta}^{+}, \\
& \frac{\partial}{\partial r} \tilde{\Theta}^{+}=-\frac{1}{2}\left(I^{\prime} / I+z R^{\prime} / I\right) \tilde{\Theta}^{+}+\left(R^{\prime} / I+z\left(R^{2} h_{1}-2 R h+h_{2}\right) / I\right) \tilde{\Theta}^{-} .
\end{aligned}
$$

It follows that

$$
\begin{aligned}
\frac{\partial}{\partial r} \tilde{P}_{2 r}^{*}= & i\left(z+u^{\prime}(r)\right) \tilde{P}_{2 r}^{*}+e^{i r z+i u(r)}\left(\frac{\partial}{\partial r} \tilde{\Theta}^{+}+i \frac{\partial}{\partial r} \tilde{\Theta}^{-}\right), \\
= & i\left(z+\frac{R^{\prime}}{2 I}\right) \tilde{P}_{2 r}^{*}-\frac{1}{2} e^{2 i u(r)}\left(\frac{I^{\prime}}{I}+z \frac{R^{\prime}}{I}\right) \tilde{P}_{2 r} \\
& -i z e^{i r z+i u(r)} I h_{1} \tilde{\Theta}^{+}+e^{i r z+i u(r)}\left(\frac{R^{\prime}}{I}+z \frac{R^{2} h_{1}-2 R h+h_{2}}{I}\right) \tilde{\Theta}^{-} .
\end{aligned}
$$

Again by Lemma 3, we have

$$
\frac{R^{2} h_{1}-2 R h+h_{2}}{I}=\frac{1}{4}\left(\frac{R^{\prime}}{I}\right)^{2} \frac{1}{I h_{1}}+\frac{1}{I h_{1}}=-K^{\prime}-I h_{1}+2,
$$


see also (39). Using this identity, we obtain

$$
\begin{aligned}
-i z I h_{1} \tilde{\Theta}^{+}+\left(\frac{R^{\prime}}{I}+z \frac{R^{2} h_{1}-2 R h+h_{2}}{I}\right) \tilde{\Theta}^{-} & =-i z I h_{1} \tilde{\Theta}^{+}+\left(\frac{R^{\prime}}{I}-z K^{\prime}-z I h_{1}+2 z\right) \tilde{\Theta}^{-} \\
& =-i z I h_{1} \tilde{E}_{r}^{\sharp}(z)+\left(\frac{R^{\prime}}{I}-z K^{\prime}+2 z\right) \tilde{\Theta}^{-} .
\end{aligned}
$$

Then relation $e^{i r z} \tilde{\Theta}^{-}=\frac{1}{2 i}\left(e^{-i u} \tilde{P}_{2 r}^{*}-e^{i u} \tilde{P}_{2 r}\right)$ implies

$$
\begin{aligned}
\frac{\partial}{\partial r} \tilde{P}_{2 r}^{*}= & i\left(z+\frac{R^{\prime}}{2 I}\right) \tilde{P}_{2 r}^{*}-\frac{1}{2} e^{2 i u(r)}\left(\frac{I^{\prime}}{I}+z \frac{R^{\prime}}{I}\right) \tilde{P}_{2 r} \\
& -i z e^{2 i u(r)} I h_{1} \tilde{P}_{2 r}+\frac{1}{2 i}\left(\frac{R^{\prime}}{I}-z K^{\prime}+2 z\right)\left(\tilde{P}_{2 r}^{*}-e^{2 i u} \tilde{P}_{2 r}\right) \\
= & \frac{i z}{2} K^{\prime} \tilde{P}_{2 r}^{*}-\frac{1}{2} e^{2 i u(r)}\left(\frac{I^{\prime}}{I}+(z-i) \frac{R^{\prime}}{I}+i z\left(2 I h_{1}+K^{\prime}-2\right)\right) \tilde{P}_{2 r} .
\end{aligned}
$$

From (39), (40) we get $2 I h_{1}+K^{\prime}-2=I^{\prime} / I$, and formula (42) follows.

Lemma 9. Let $D_{\mu}$ be the Szegố function of $\mu$. Then $\lim _{r \rightarrow+\infty} \tilde{P}_{r}^{*}(z)=D_{\mu}^{-1}(z)$ for every $z \in \mathbb{C}^{+}$. Moreover, $\lim _{r \rightarrow+\infty} \tilde{P}_{r}(z)=0$ and $\int_{\mathbb{R}_{+}}\left|\tilde{P}_{r}(z)\right|^{2} d r<+\infty$ for $z \in \mathbb{C}^{+}$.

Proof. Fix $z \in \mathbb{C}^{+}$. By Lemma 7 and Lemma 8 , we have

$$
\frac{\partial}{\partial r} \tilde{P}_{r}^{*}(z)=f_{1}(r) \tilde{P}_{r}(z)+f_{2}(r) \tilde{P}_{r}^{*}(z),
$$

for some functions $f_{1} \in L^{1}\left(\mathbb{R}_{+}\right)+L^{2}\left(\mathbb{R}_{+}\right), f_{2} \in L^{1}\left(\mathbb{R}_{+}\right)$depending on $z$. It follows that

$$
\begin{aligned}
\frac{\partial}{\partial r}\left|\tilde{P}_{r}^{*}(z)\right|^{2} & =2 \operatorname{Re}\left(\overline{\tilde{P}_{r}^{*}(z)} \frac{\partial}{\partial r} \tilde{P}_{r}^{*}(z)\right), \\
& =2 \operatorname{Re}\left(f_{1}(r) \tilde{P}_{r}(z) \overline{\tilde{P}_{r}^{*}(z)}+f_{2}(r)\left|\tilde{P}_{r}^{*}(z)\right|^{2}\right), \\
& =2 \operatorname{Re}\left(f_{1}(r) \tilde{P}_{r}(z) \overline{\tilde{P}_{r}^{*}(z)}\right)-2 \operatorname{Re} f_{2}(r)\left|\tilde{P}_{r}^{*}(z)\right|^{2} .
\end{aligned}
$$

Since $\left|\tilde{P}_{r}(z)\right|^{2}=e^{-2 r \operatorname{Im} z}\left|\tilde{P}_{r}^{*}(\bar{z})\right|^{2}$, the above formula for $\bar{z}$ implies

$$
\begin{aligned}
\frac{\partial}{\partial r}\left|\tilde{P}_{r}(z)\right|^{2}= & -2 \operatorname{Im} z\left|\tilde{P}_{r}(z)\right|^{2}+2 e^{-2 r \operatorname{Im} z} \operatorname{Re}\left(g_{1}(r) \tilde{P}_{r}(\bar{z}) \overline{\tilde{P}_{r}^{*}(\bar{z})}\right) \\
& +2 \operatorname{Re} g_{2}(r) e^{-2 r \operatorname{Im} z}\left|\tilde{P}_{r}^{*}(\bar{z})\right|^{2},
\end{aligned}
$$

for some functions $g_{1} \in L^{1}\left(\mathbb{R}_{+}\right)+L^{2}\left(\mathbb{R}_{+}\right), g_{2} \in L^{1}\left(\mathbb{R}_{+}\right)$. Since $e^{-i r \bar{z}} \tilde{P}_{r}(\bar{z})=\overline{\tilde{P}_{r}^{*}(z)}$ and $e^{i r z} \overline{\tilde{P}_{r}^{*}(\bar{z})}=\tilde{P}_{r}(z)$, we have

$$
\frac{\partial}{\partial r}\left|\tilde{P}_{r}(z)\right|^{2}=-2 \operatorname{Im} z\left|\tilde{P}_{r}(z)\right|^{2}+2 \operatorname{Re}\left(g_{1}(r) \tilde{P}_{r}(z) \overline{\tilde{P}_{r}^{*}(z)}\right)+2 \operatorname{Re} g_{2}(r)\left|\tilde{P}_{r}(z)\right|^{2} .
$$

It follows that

$$
\begin{aligned}
\frac{\partial}{\partial r}\left|\tilde{P}_{r}^{*}(z)\right|^{2}-\frac{\partial}{\partial r}\left|\tilde{P}_{r}(z)\right|^{2} \geqslant & 2 \operatorname{Im} z\left|\tilde{P}_{r}(z)\right|^{2}-h_{1}(r)\left|\tilde{P}_{r}(z) \tilde{P}_{r}^{*}(z)\right| \\
& -h_{2}(r)\left(\left|\tilde{P}_{r}(z)\right|^{2}+\left|\tilde{P}_{r}^{*}(z)\right|^{2}\right),
\end{aligned}
$$

for some positive functions $h_{1} \in L^{1}\left(\mathbb{R}_{+}\right)+L^{2}\left(\mathbb{R}_{+}\right), h_{2} \in L^{1}\left(\mathbb{R}_{+}\right)$. As we have seen in Section 2.3, the function

$$
k_{2 r, \lambda}: z \mapsto-\frac{1}{2 \pi i} \frac{\tilde{P}_{r}^{*}(z) \overline{\tilde{P}_{r}^{*}(\lambda)}-\tilde{P}_{r}(z) \overline{\tilde{P}_{r}(\lambda)}}{z-\bar{\lambda}}, \quad z \in \mathbb{C},
$$

is the reproducing kernel of the space $\left(\mathrm{PW}_{[0, r]}, \mu\right)$ at $\lambda \in \mathbb{C}$. Hence for every $z \in \mathbb{C}^{+}$ the absolutely continuous function $\psi: r \mapsto\left|\tilde{P}_{r}^{*}(z)\right|^{2}-\left|\tilde{P}_{r}(z)\right|^{2}$ increases and does not exceed $\left|D_{\mu}^{-1}(z)\right|^{2}$, where $D_{\mu}$ is the Szegó function (3) of $\mu$. In particular, we 
have $\psi^{\prime} \in L^{1}\left(\mathbb{R}_{+}\right)$. Since $\left|\tilde{P}_{0}(z)\right|>0$ and $\tilde{P}_{r}(z)$ is continuous in $r$, there exists a number $r_{0}>0$ such that $\min _{0 \leqslant r \leqslant r_{0}}\left|\tilde{P}_{r}(z)\right|^{2}>0$. On the other hand, for $r>r_{0}$ we have $0<\psi\left(r_{0}\right) \leqslant \psi(r) \leqslant\left|\tilde{P}_{r}^{*}(z)\right|^{2}$. Hence there is a constant $c>0$ such that $\left|\tilde{P}_{r}^{*}(z)\right|^{2} \geqslant c$ for every $r \geqslant 0$. Denote $\tilde{h}_{1,2}=h_{1,2} / 2 \operatorname{Im} z$. By the Hermite-Biehler property of $\tilde{E}_{r}$, we have $\left|\tilde{P}_{r}(z) / \tilde{P}_{r}^{*}(z)\right| \leqslant 1$ for all $r \geqslant 0$. From (47) we get

$$
\frac{\left|\tilde{P}_{r}(z)\right|^{2}}{\left|\tilde{P}_{r}^{*}(z)\right|^{2}} \leqslant \frac{\psi^{\prime}(r)}{2 c \operatorname{Im} z}+\tilde{h}_{1}(r)\left|\frac{\tilde{P}_{r}(z)}{\tilde{P}_{r}^{*}(z)}\right|+2 \tilde{h}_{2}(r) .
$$

Consider the set $S=\left\{r \in \mathbb{R}_{+}:\left|\tilde{h}_{1}(r)\right| \geqslant 1\right\}$. We have $\tilde{h}_{1} \in L^{1}(S), \tilde{h}_{1} \in L^{2}\left(\mathbb{R}_{+} \backslash S\right)$. Recall also that $\tilde{h}_{2} \in L^{1}\left(\mathbb{R}_{+}\right)$and $\psi^{\prime} \in L^{1}\left(\mathbb{R}_{+}\right)$. Formula (49), Cauchy inequality, and the bound $\left|\tilde{P}_{r}(z) / \tilde{P}_{r}^{*}(z)\right| \leqslant 1$ imply the existence of a new constant $c>0$ such that

$$
\begin{aligned}
\int_{0}^{t} \frac{\left|\tilde{P}_{r}(z)\right|^{2}}{\left|\tilde{P}_{r}^{*}(z)\right|^{2}} d r & \leqslant c+\left\|\tilde{h}_{1}\right\|_{L^{1}(S)}+\left\|\tilde{h}_{1}\right\|_{L^{2}\left(\mathbb{R}_{+} \backslash S\right)}\left(\int_{0}^{t} \frac{\left|\tilde{P}_{r}(z)\right|^{2}}{\left|\tilde{P}_{r}^{*}(z)\right|^{2}} d r\right)^{\frac{1}{2}} \\
& \leqslant c+c\left(\int_{0}^{t} \frac{\left|\tilde{P}_{r}(z)\right|^{2}}{\left|\tilde{P}_{r}^{*}(z)\right|^{2}} d r\right)^{\frac{1}{2}}
\end{aligned}
$$

for every $t>0$. It follows that

$$
\frac{\tilde{P}_{r}(z)}{\tilde{P}_{r}^{*}(z)} \in L^{2}\left(\mathbb{R}_{+}\right) \cap L^{\infty}\left(\mathbb{R}_{+}\right) .
$$

Next, formula (42) for $z=i$ takes the form $\frac{\partial}{\partial r} \log \tilde{P}_{2 r}^{*}(i)=-K^{\prime}(r) / 2$. We have $K^{\prime} \leqslant 0$ almost everywhere on $\mathbb{R}_{+}$by Lemma 2. Since $\tilde{P}_{0}^{*}(i)=1 / \sqrt{I(0)}$ is positive by construction, we see that $\tilde{P}_{r}^{*}(i)>0$ for all $r>0$. Then Lemma 6 and Theorem 2 imply

$$
\lim _{r \rightarrow+\infty} \tilde{P}_{r}^{*}(z)=D_{\mu}^{-1}(z), \quad z \in \mathbb{C}^{+} .
$$

Indeed, the functions $\tilde{P}_{r}^{*}, D_{\mu}^{-1}$ are outer in $\mathbb{C}^{+}$, take positive values at $z=i$, and $\log \left|\tilde{P}_{r}^{*}\right|$ tends to $\log \left|D_{\mu}^{-1}\right|$ in $L^{1}(P)$ as $r \rightarrow+\infty$ by Theorem 2. Formulas (50) and (51) give us $\left|\tilde{P}_{r}(z)\right|^{2} \in L^{1}\left(\mathbb{R}_{+}\right) \cap L^{\infty}\left(\mathbb{R}_{+}\right)$. Moreover, we have $\frac{\partial}{\partial r}\left|\tilde{P}_{r}(z)\right|^{2} \in L^{1}\left(\mathbb{R}_{+}\right)$ by (46). It follows that $\lim _{r \rightarrow+\infty} \tilde{P}_{r}(z)=0$ for every $z \in \mathbb{C}^{+}$.

Next lemma is similar to Corollary 5.10 in [8].

Lemma 10. We have $\lim _{r \rightarrow+\infty}\left\|\tilde{P}_{r} /(x+i)\right\|_{L^{2}\left(\mu_{\mathrm{s}}\right)}=0$. Moreover if a set $E$ is such that $|\mathbb{R} \backslash E|=0, \mu_{\mathbf{s}}(E)=0$, then $\lim _{r \rightarrow+\infty}\left\|\left(\tilde{P}_{r}^{*}-\chi_{E} D_{\mu}^{-1}\right) /(x+i)\right\|_{L^{2}(\mu)}=0$.

Proof. Fix the set $E$ from the statement of the lemma. From Theorem 2 and Jensen inequality we get

$$
\begin{aligned}
1 & =\lim _{r \rightarrow \infty} \exp \left(\int_{\mathbb{R}} \log \left(\left|\tilde{P}_{r}(x)\right|^{2} w(x)\right) d P(x)\right) \leqslant \limsup _{r \rightarrow \infty} \int_{\mathbb{R}}\left|\tilde{P}_{r}(x)\right|^{2} w(x) d P(x) \\
& \leqslant \limsup _{r \rightarrow \infty} \frac{1}{\pi} \int_{\mathbb{R}} \frac{\chi_{E}(x)\left|\tilde{P}_{r}(x)\right|^{2}}{1+x^{2}} d \mu(x) \leqslant \limsup _{r \rightarrow \infty} \frac{1}{\pi} \int_{\mathbb{R}} \frac{\left|\tilde{P}_{r}(x)\right|^{2}}{1+x^{2}} d \mu(x) .
\end{aligned}
$$


Recall that the reproducing kernel of the space $\left(\mathrm{PW}_{[0, r]}, \mu\right)$ is given by (48). By Lemma 9, we have

$$
\begin{aligned}
\limsup _{r \rightarrow \infty}\left\|\frac{\tilde{P}_{r}^{*}}{x+i}\right\|_{L^{2}(\mu)}^{2} & =\limsup _{r \rightarrow \infty} \frac{1}{\left|\tilde{P}_{r}^{*}(i)\right|^{2}}\left\|\frac{\tilde{P}_{r}^{*}(z) \overline{\tilde{P}_{r}^{*}(i)}-\tilde{P}_{r}(z) \overline{\tilde{P}_{r}(i)}}{z+i}\right\|_{L^{2}(\mu)}^{2} \\
& =\pi \limsup _{r \rightarrow \infty} \frac{\left|\tilde{P}_{r}^{*}(i)\right|^{2}-\left|\tilde{P}_{r}(i)\right|^{2}}{\left|\tilde{P}_{r}^{*}(i)\right|^{2}}=\pi .
\end{aligned}
$$

Since $\left|\tilde{P}_{r}^{*}\right|=\left|\tilde{P}_{r}\right|$ on $\mathbb{R}$, from here we see that inequalities in (52) are, in fact, equalities. It follows that

$$
\lim _{r \rightarrow+\infty}\left\|\tilde{P}_{r} /(x+i)\right\|_{L^{2}\left(\mu_{\mathbf{s}}\right)}=0, \quad \lim _{r \rightarrow+\infty}\left\|\tilde{P}_{r} D_{\mu}\right\|_{L^{2}(d P)}^{2}=1 .
$$

Next, by the mean value theorem for the harmonic function $\operatorname{Re}\left(\tilde{P}_{r}^{*} D_{\mu}\right)$, we have

$$
\begin{aligned}
\frac{1}{\pi} \int_{\mathbb{R}}\left|\frac{\tilde{P}_{r}^{*}(x)}{x+i}-\frac{D_{\mu}(x)^{-1}}{x+i}\right|^{2}|D(x)|^{2} d x & =\int_{\mathbb{R}}\left|\tilde{P}_{r}^{*}(x) D_{\mu}(x)-1\right|^{2} d P(x), \\
& =\left\|\tilde{P}_{r} D_{\mu}\right\|_{L^{2}(d P)}^{2}+1-2 \operatorname{Re}\left(\tilde{P}_{r}^{*}(i) D_{\mu}(i)\right) .
\end{aligned}
$$

By Lemma 9 and (54), the right hand side of the above formula converges to zero as $r \rightarrow+\infty$. Using the fact that $\mu=\left|D_{\mu}(x)\right|^{2} d x+\mu_{\mathbf{s}}$ and the first relation in (54), we complete the proof of the lemma.

\section{Proof of Theorem 1}

We will use the following simple lemma, see, e.g, [4]

Lemma 11. For every continuous function $f$ with compact support on $\mathbb{R}_{+}$and for all $t>0$ large enough we have

$$
e^{-i t \mathcal{D}_{0}}\left(\begin{array}{l}
f \\
0
\end{array}\right)=\frac{1}{2}\left(\begin{array}{c}
f(|s-t|) \\
i f(|s-t|)
\end{array}\right), \quad e^{-i t \mathcal{D}_{0}}\left(\begin{array}{l}
0 \\
f
\end{array}\right)=-\frac{i}{2}\left(\begin{array}{c}
\operatorname{sign}(s-t) f(|s-t|) \\
i \operatorname{sign}(s-t) f(|s-t|)
\end{array}\right),
$$

for all $s \in \mathbb{R}_{+}$.

Proof of Theorem 1, Let $q_{1}, q_{2}$ be real functions on $\mathbb{R}_{+}$such that $q_{1,2} \in L_{\text {loc }}^{1}\left(\mathbb{R}_{+}\right)$. Consider the Dirac operator $\mathcal{D}_{Q}$ with the potential $Q=\left(\begin{array}{cc}q_{1} & q_{2} \\ q_{2} & -q_{1}\end{array}\right)$. Assume that the spectral measure $\mu$ of $\mathcal{D}_{Q}$ belongs to the Szegó class $\mathrm{Sz}(\mathbb{R})$. Using construction from Section 2.4, define the Hamiltonian $\mathcal{H}=N_{0}^{*} N_{0}$ on $\mathbb{R}_{+}$such that $\operatorname{det} \mathcal{H}=1$ and the spectral measure of $\mathcal{H}$ coincides with $\mu$. Let $I=\mathcal{I}_{\mathcal{H}}, R=\mathcal{R}_{\mathcal{H}}, K=\mathcal{K}_{\mathcal{H}}$ be the functions from Section 2.2 and let $G$ be defined by (43). Consider the densely defined multiplication operator on $L^{2}\left(\mathbb{R}_{+}, \mathbb{C}^{2}\right)$ taking a continuous compactly supported vector-function $X \in L^{2}\left(\mathbb{R}_{+}, \mathbb{C}^{2}\right)$ into $N X$, where $N=J N_{0} J^{*} G^{*} \Sigma_{u}$,

$$
\Sigma_{u}(r)=\left(\begin{array}{cc}
\cos u(r) & \sin u(r) \\
-\sin u(r) & \cos u(r)
\end{array}\right), \quad u(r)=\frac{\mathcal{R}_{\mathcal{H}}^{\prime}(r)}{2 \mathcal{I}_{\mathcal{H}}(r)}, \quad r \geqslant 0 .
$$

With a slight abuse of notation, we will denote this operator by the same letter $N$. Let $\Psi$ be the solution of (5), and let $\Theta$ be defined by (11), $\tilde{\Theta}=G \Theta$. Since $N_{0}$ is a real matrix with $\operatorname{det} N_{0}=1$, we have $N^{-1}=J N_{0}^{*} J^{*}$. Using relation $\Psi=N_{0} \Theta$ from Section 2.4, we obtain

$$
\langle N(r) e, \Psi(r, x)\rangle_{\mathbb{C}^{2}}=\left\langle e, \Sigma_{u}^{*}(r) G(r) N_{0}^{-1}(r) \Psi(r, x)\right\rangle_{\mathbb{C}^{2}}=\left\langle e, \Sigma_{u}^{*}(r) \tilde{\Theta}(r, x)\right\rangle_{\mathbb{C}^{2}},
$$

\footnotetext{
${ }^{1}$ It worth be mentioned that $D_{0}=-\mathcal{D}_{0}$ for the free Dirac operator $D_{0}$ used in [4. In particular, we have $e^{i t \mathcal{D}_{0}}=e^{-i t D_{0}}$ for all $t \in \mathbb{R}$.
} 
for every vector $e \in \mathbb{C}^{2}$ and all $r \geqslant 0, x \in \mathbb{R}$. Define $\tilde{P}_{2 r}, \tilde{P}_{2 r}^{*}$ by (37). Consider a smooth function $f$ on $\mathbb{R}_{+}$supported on $(0, a), a>0$. Lemma 11 and the spectral theorem for $\mathcal{D}_{Q}$ yield

$$
\begin{aligned}
\mathcal{F}_{Q} e^{i t \mathcal{D}_{Q}} N e^{-i t \mathcal{D}_{0}}\left(\begin{array}{l}
f \\
0
\end{array}\right) & =\frac{1}{\sqrt{\pi}} \int_{\mathbb{R}_{+}}\left\langle e^{i t \mathcal{D}_{Q}} N e^{-i t \mathcal{D}_{0}}\left(\begin{array}{c}
f \\
0
\end{array}\right), \Psi(s, x)\right\rangle_{\mathbb{C}^{2}} d s, \\
& =\frac{e^{i t x}}{2 \sqrt{\pi}} \int_{\mathbb{R}_{+}}\left\langle N(s)\left(\begin{array}{c}
f(|s-t|) \\
i f(|s-t|)
\end{array}\right), \Psi(s, x)\right\rangle_{\mathbb{C}^{2}} d s, \\
& =\frac{e^{i t x}}{2 \sqrt{\pi}} \int_{\mathbb{R}_{+}} f(|s-t|)\left\langle\Sigma_{u}(s)\left(\begin{array}{c}
1 \\
i
\end{array}\right), \tilde{\Theta}(s, x)\right\rangle_{\mathbb{C}^{2}} d s, \\
& =\frac{e^{i t x}}{2 \sqrt{\pi}} \int_{\mathbb{R}_{+}} f(|s-t|) e^{-i x s} \tilde{P}_{2 s}^{*}(x) d s,
\end{aligned}
$$

for $t>0$ big enough and all $x \in \mathbb{R}$. Next, expression $e^{i x t} \int_{\mathbb{R}_{+}} f(|s-t|) e^{-i x s} \tilde{P}_{2 s}^{*}(x) d s$ for $t>a$ equals

$$
\begin{aligned}
\int_{-a}^{a} f & (|s|) e^{-i x s} \tilde{P}_{2 s+2 t}^{*}(x) d s=\int_{-a}^{a}\left[\int_{-a}^{s} f(|\tau|) e^{-i x \tau} d \tau\right]^{\prime} \tilde{P}_{2 s+2 t}^{*}(x) d s \\
& =\tilde{P}_{2 a+2 t}^{*}(x) \int_{-a}^{a} f(|\tau|) e^{-i x \tau} d \tau+\int_{-a}^{a} \frac{\partial}{\partial s} \tilde{P}_{2 s+2 t}^{*}(x) \int_{-a}^{s} f(|\tau|) e^{-i x \tau} d \tau d s .
\end{aligned}
$$

Denote the second summand in the last formula by $h_{t}(x)$. Let us show that $\lim _{t \rightarrow+\infty}\left\|h_{t}\right\|_{L^{2}(\mu)}=0$. Since $f$ is smooth, we have

$$
\sup _{s \in(-a, a)}\left|\hat{f}_{c}(x, s)\right| \leqslant \frac{c}{(1+|x|)^{2}}, \quad \hat{f}_{c}(x, s)=\int_{-a}^{s} f(|\tau|) e^{-i x \tau} d \tau,
$$

for all $x \in \mathbb{R}$ and a constant $c$ depending only on $f$. Recall that $|\tilde{P}|=\left|\tilde{P}^{*}\right|$ on $\mathbb{R}_{+}$ by construction. By Lemma 8 there exists a function $g \in L^{1}(\mathbb{R})+L^{2}\left(\mathbb{R}_{+}\right)$such that $\left|\frac{\partial}{\partial r} \tilde{P}_{r}^{*}(x)\right| \leqslant(1+|x|) g(x)\left|\tilde{P}_{r}(x)\right|$ for $x \in \mathbb{R}$. Hence one can use the generalized Minkowski inequality to estimate

$$
\begin{aligned}
\left\|h_{t}\right\|_{L^{2}(\mu)}^{2} & \leqslant c \int_{\mathbb{R}}\left(\int_{-a}^{a} g(s+t) \frac{\left|\tilde{P}_{s+t}^{*}(x)\right|}{1+|x|} d s\right)^{2} d \mu(x) \\
& \leqslant c\left(\int_{-a}^{a} g(s+t) d s\right)^{2} \sup _{s \in[-a, a]} \int_{\mathbb{R}} \frac{\left|\tilde{P}_{s+t}^{*}(x)\right|^{2}}{(1+|x|)^{2}} d \mu(x) .
\end{aligned}
$$

Formula (53) gives $\lim \sup _{r \rightarrow \infty} \int_{\mathbb{R}} \frac{\left|\tilde{P}_{r}^{*}(x)\right|^{2}}{(1+|x|)^{2}} d \mu<+\infty$, hence $\lim _{t \rightarrow \infty}\left\|h_{t}\right\|_{L^{2}(\mu)}=0$. By Lemma10, functions $\tilde{P}_{2 a+2 t}^{*} \int_{-a}^{a} f(|\tau|) e^{-i x \tau} d \tau$ tend to $\chi_{E} D_{\mu}^{-1} \int_{-a}^{a} f(|\tau|) e^{-i x \tau} d \tau$ in $L^{2}(\mu)$ as $t \rightarrow+\infty$. Hence there exists the strong limit in $L^{2}(\mu)$

$$
\lim _{r \rightarrow+\infty} \mathcal{F}_{Q} e^{i t \mathcal{D}_{Q}} N e^{-i t \mathcal{D}_{0}}\left(\begin{array}{l}
f \\
0
\end{array}\right)=\frac{\chi_{E} D_{\mu}^{-1}}{\sqrt{\pi}} \int_{0}^{\infty} f(t) \cos x t d t=\chi_{E} D_{\mu}^{-1} \mathcal{F}_{0}\left(\begin{array}{l}
f \\
0
\end{array}\right) .
$$

Similar arguments give the formula

$$
\lim _{r \rightarrow+\infty} \mathcal{F}_{Q} e^{i t \mathcal{D}_{Q}} N e^{-i t \mathcal{D}_{0}}\left(\begin{array}{l}
0 \\
f
\end{array}\right)=-\frac{\chi_{E} D_{\mu}^{-1}}{\sqrt{\pi}} \int_{0}^{\infty} f(t) \sin x t d t=\chi_{E} D_{\mu}^{-1} \mathcal{F}_{0}\left(\begin{array}{l}
0 \\
f
\end{array}\right),
$$


for smooth compactly supported functions $f$. Our aim now is to replace $N$ by the operator $X \mapsto \Sigma_{\varphi} X$ for some function $\varphi$. Let us define $\varphi$ on $\mathbb{R}_{+}$so that $\tilde{N}(r)=\Sigma_{\varphi(r)} N(r)$ is positive definite for every $r \geqslant 0$. We are going to show that

$$
\lim _{t \rightarrow+\infty}\left\|\tilde{N} S_{t} X-S_{t} X\right\|_{L^{2}\left(\mathbb{R}_{+}, \mathbb{C}^{2}\right)}=0, \quad\left(S_{t} X\right)(s)=X(t+s),
$$

for every continuous vector-function $X$ with compact support. By construction, we have $\operatorname{det} \tilde{N}=1$ on $\mathbb{R}_{+}$. For $s \geqslant 0$, let $e_{1}(s), e_{2}(s)$ be the unit eigenvectors of the matrix $\tilde{N}(s)$ corresponding to the eigenvalues $\lambda(s), 1 / \lambda(s)$, correspondingly. Then for every vector $e=c_{1} e_{1}(r)+c_{2} e_{2}(r)$ in $\mathbb{C}^{2}$ we have

$$
\|N e-e\|_{\mathbb{C}^{2}}^{2}=\left|c_{1}\right|^{2}(\lambda(s)-1)^{2}+\left|c_{2}\right|^{2}(1 / \lambda(s)-1)^{2} \leqslant(\lambda(s)-1 / \lambda(s))^{2}\|e\|_{\mathbb{C}^{2}}^{2},
$$

where we used twice the elementary inequality $(x-1)^{2} \leqslant(x-1 / x)^{2}$ for $x>0$. Note that

$$
\begin{aligned}
(\lambda(s)-1 / \lambda(s))^{2} & =\operatorname{trace} \tilde{N}^{2}(s)-2=\operatorname{trace} N^{*}(s) N(s)-2 \\
& =\operatorname{trace} G(s) J \mathcal{H}(s) J^{*} G^{*}(s)-2
\end{aligned}
$$

A calculation gives

$$
G J \mathcal{H} J^{*} G^{*}=\left(\begin{array}{cc}
\frac{h_{2}-2 R h+R^{2} h_{1}}{I I} & R h_{1}-h \\
R h_{1}-h & I h_{1}
\end{array}\right)=\left(\begin{array}{cc}
1 & 0 \\
0 & 1
\end{array}\right)+\left(\begin{array}{cc}
\frac{1}{I h_{1}}-1+\frac{u^{2}}{I h_{1}} & u \\
u & I h_{1}-1
\end{array}\right) .
$$

From Lemma[7 we see that $g=\operatorname{trace} G J \mathcal{H} J^{*} G^{*}-2$ is a positive function in $L^{1}\left(\mathbb{R}_{+}\right)$. Hence (56) holds:

$$
\limsup _{t \rightarrow+\infty}\left\|\tilde{N} S_{t} X-S_{t} X\right\|_{L^{2}\left(\mathbb{R}_{+}, \mathbb{C}^{2}\right)}^{2} \leqslant \limsup _{t \rightarrow+\infty} \int_{0}^{\infty} g(s)\|X(t+s)\|_{\mathbb{C}^{2}}^{2} d s=0 .
$$

It follows that for every smooth function $X$ with compact support the strong $L^{2}(\mu)$ -

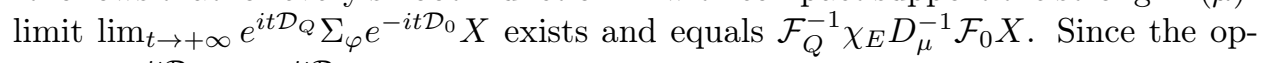
erators $e^{i t \mathcal{D}_{Q} \Sigma_{\varphi} e^{-i t \mathcal{D}_{0}}}$ are unitary, this implies the existence of the limit

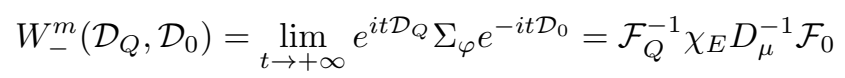

in the strong operator topology. Existence of the wave operator

$$
W_{+}^{m}\left(\mathcal{D}_{Q}, \mathcal{D}_{0}\right)=\mathcal{F}_{Q}^{-1} \chi_{E} \overline{D_{\mu}^{-1}} \mathcal{F}_{0}
$$

can be proved in a similar way. In the case where $q_{1}=0$, the Hamiltonian $\mathcal{H}$ generated by $Q$ has the diagonal form, see Section 2.4 In particular, we have $\mathcal{R}_{\mathcal{H}}=0$ by Lemma 2.2 in [1]. Hence our construction gives $u=\varphi=0, \Sigma_{\varphi}=\left(\begin{array}{ll}1 & 0 \\ 0 & 1\end{array}\right)$, $W_{ \pm}^{m}\left(\mathcal{D}_{Q}, \mathcal{D}_{0}\right)=W_{ \pm}\left(\mathcal{D}_{Q}, \mathcal{D}_{0}\right)$ in this case. The theorem follows.

Corollary 1 follows immediately from Theorem 1 and the following proposition.

Proposition 2. Let $q \in L_{\text {loc }}^{1}\left(\mathbb{R}_{+}\right)$be a real function on $\mathbb{R}_{+}$, and let $Q=\left(\begin{array}{cc}0 & q \\ q & 0\end{array}\right)$. Then the spectral measure of $\mathcal{D}_{Q}$ belongs to $\mathrm{Sz}(\mathbb{R})$ if and only if $q$ satisfies (8).

Proof. Consider the Hamiltonian

$$
\mathcal{H}(t)=\left(\begin{array}{cc}
e^{-2 g(t)} & 0 \\
0 & e^{2 g(t)}
\end{array}\right), \quad g(t)=\int_{0}^{t} q(s) d s,
$$

on $\mathbb{R}_{+}$. Condition (8) for $q$ is equivalent to the condition (15) for $\mathcal{H}$. By Theorem 3 , the spectral measure of $\mathcal{H}$ belongs to $\mathrm{Sz}(\mathbb{R})$. It remains to use the fact that the spectral measures of $\mathcal{H}$ and $\mathcal{D}_{Q}$ coincide, see Section 2.4. 


\section{Appendix}

Here we prove Lemmas 1, 2, and 3 following the ideas of [1].

Proof of Lemma 1. Assertions $(a),(b)$ of Lemma 1 are the formulas (2.13), (2.14) in [1], correspondingly.

Proof of Lemma 2. A straightforward calculation shows that the Weyl function of the constant nontrivial Hamiltonian

$$
\mathcal{H}=\left(\begin{array}{cc}
c_{1} & c \\
c & c_{2}
\end{array}\right)
$$

equals $m=i c_{1}^{-1} \sqrt{c_{1} c_{2}-c^{2}}+c / c_{1}$. Now let $\mathcal{H}$ be an arbitrary singular nontrivial Hamiltonian and let $\widehat{\mathcal{H}}_{r}$ be defined by (16). Then the Weyl function of $\left(\hat{\mathcal{H}}_{r}\right)_{r}$ is

$$
m_{\left(\hat{\mathcal{H}}_{r}\right)_{r}}=i \mathcal{I}_{\mathcal{H}}(r)+\mathcal{R}_{\mathcal{H}}(r) .
$$

Hence, we have $\mathcal{J}_{\widehat{\mathcal{H}}_{r}}(r)=\log c_{1}^{-1} \sqrt{c_{1} c_{2}-c^{2}}=-\log c_{1}=\log \mathcal{I}_{\mathcal{H}}(r)$. Next, let $F_{r}, G_{r}$ and $\hat{F}_{r}, \hat{G}_{r}$ be the functions from Lemma 1 for the Hamiltonians $\mathcal{H}$ and $\hat{\mathcal{H}}_{r}$, correspondingly. Note that $F_{r}(i)=\hat{F}_{r}(i), G_{r}(i)=\hat{G}_{r}(i)$ by construction and formula (57). It follows from assertion $(a)$ of Lemma 1 that $\hat{m}_{r}(i)=m_{0}(i)$, that is,

$$
\mathcal{I}_{\widehat{\mathcal{H}}_{r}}(0)=\mathcal{I}_{\mathcal{H}}(0), \quad \mathcal{R}_{\widehat{\mathcal{H}}_{r}}(0)=\mathcal{R}_{\mathcal{H}}(0) .
$$

As in the proof of Lemma 2.5 in [1], we have

$$
\mathcal{J}_{\mathcal{H}}(r)=\mathcal{J}_{\mathcal{H}}(0)-2 \xi_{\mathcal{H}}(r)+2 \log \left|F_{r}(i)\right|,
$$

where $\xi_{\mathcal{H}}: r \mapsto \int_{0}^{r} \sqrt{\operatorname{det} \mathcal{H}(t)} d t$. Similarly,

$$
\mathcal{J}_{\widehat{\mathcal{H}}_{r}}(r)=\mathcal{J}_{\widehat{\mathcal{H}}_{r}}(0)-2 \xi_{\widehat{\mathcal{H}}_{r}}(r)+2 \log \left|\hat{F}_{r}(i)\right| \text {. }
$$

Since $\xi_{\mathcal{H}}(r)=\xi_{\widehat{\mathcal{H}}_{r}}(r)$ and $\hat{F}_{r}(i)=F_{r}(i)$, we have

$$
\begin{aligned}
\mathcal{K}_{\mathcal{H}}(r) & =\log \mathcal{I}_{\mathcal{H}}(r)-\mathcal{J}_{\mathcal{H}}(r)=\mathcal{J}_{\widehat{\mathcal{H}}_{r}}(r)-\mathcal{J}_{\mathcal{H}}(r) \\
& =\mathcal{J}_{\widehat{\mathcal{H}}_{r}}(0)-\mathcal{J}_{\mathcal{H}}(0)=\mathcal{J}_{\widehat{\mathcal{H}}_{r}}(0)-\log \mathcal{I}_{\widehat{\mathcal{H}}_{r}}(0)+\log \mathcal{I}_{\mathcal{H}}(0)-\mathcal{J}_{\mathcal{H}}(0), \\
& =-\mathcal{K}_{\widehat{\mathcal{H}}_{r}}(0)+\mathcal{K}_{\mathcal{H}}(0) .
\end{aligned}
$$

The last formula can be rewritten in the form $\mathcal{K}_{\mathcal{H}}(0)=\mathcal{K}_{\mathcal{H}}(r)+\mathcal{K}_{\widehat{\mathcal{H}}_{r}}(0)$. Since the functions $\mathcal{K}_{\mathcal{H}}, \mathcal{K}_{\widehat{\mathcal{H}}_{r}}$ are nonnegative, we see that $\mathcal{K}_{\mathcal{H}}$ is nonincreasing. This fact and the semi-continuity of logarithmic integrals implies $\lim _{r \rightarrow+\infty} \mathcal{K}_{\widehat{\mathcal{H}}_{r}}(0)=\mathcal{K}_{\mathcal{H}}(0)$, or, equivalently, $\lim _{r \rightarrow+\infty} \mathcal{K}_{\mathcal{H}}(r)=0$, see details in Lemma 4.1 of [1].

Proof of Lemma 3. Assume first that the Hamiltonian $\mathcal{H}=\left(\begin{array}{cc}h_{1} & h \\ h & h_{2}\end{array}\right)$ is continuously differentiable on $\mathbb{R}_{+}$. As in the proof of Lemma 2.7 in [1], formula (5) yields

$$
\left.\left(\begin{array}{cc}
\Theta^{+}(r, i)^{\prime} & \Phi^{+}(r, i)^{\prime} \\
\Theta^{-}(r, i)^{\prime} & \Phi^{-}(r, i)^{\prime}
\end{array}\right)\right|_{r=0}=\left(\begin{array}{cc}
i h(0) & i h_{2}(0) \\
-i h_{1}(0) & -i h(0)
\end{array}\right) .
$$

Then (58) and the initial condition $M(0, i)=\left(\begin{array}{ll}1 & 0 \\ 0 & 1\end{array}\right)$ give

$$
\begin{aligned}
\mathcal{J}_{\mathcal{H}}^{\prime}(0) & =-2 \xi^{\prime}(0)+\left.2 \operatorname{Re}\left(\frac{\Theta^{+}(r, i)^{\prime}+m_{r}^{\prime}(i) \Theta^{-}(r, i)+m_{r}(i) \Theta^{-}(r, i)^{\prime}}{\Theta^{+}(r, i)+m_{r}(i) \Theta^{-}(r, i)}\right)\right|_{r=0}, \\
& =-2 \xi^{\prime}(0)+2 \operatorname{Re}\left(i h(0)-i m_{0}(i) h_{1}(0)\right), \\
& =-2 \xi^{\prime}(0)+2 h_{1}(0) \mathcal{I}_{\mathcal{H}}(0),
\end{aligned}
$$


where the derivatives are taken with respect to $r$. The same relation for the Hamiltonian $\mathcal{H}_{r}$ in place of $\mathcal{H}$ shows that $\mathcal{J}_{\mathcal{H}}^{\prime}(r)=-2 \xi_{\mathcal{H}}^{\prime}(r)+2 h_{1}(r) \mathcal{I}_{\mathcal{H}}(r)$ for all $r>0$. Similarly, differentiating relation $m_{0}(i)=\frac{G_{r}(i)}{F_{r}(i)}$ from Lemma 1 at $r=0$ and using (59), we obtain $0=i h_{2}(0)+m_{0}^{\prime}(i)-i m_{0}(i) h(0)-m_{0}(i)\left(i h(0)-i m_{0}(i) h_{1}(0)\right)$. As before, this gives

$$
0=i h_{2}(r)+m_{r}^{\prime}(i)-i m_{r}(i) h(r)-m_{r}(i)\left(i h(r)-i m_{r}(i) h_{1}(r)\right)
$$

for all $r>0$. By construction, we have $m_{r}(i)=i \mathcal{I}_{\mathcal{H}}(r)+\mathcal{R}_{\mathcal{H}}(r)$. Taking imaginary and real parts in (60), we obtain

$$
\begin{aligned}
& 0=h_{2}+\mathcal{I}_{\mathcal{H}}^{\prime}-2 \mathcal{R}_{\mathcal{H}} h+\mathcal{R}_{\mathcal{H}}^{2} h_{1}-\mathcal{I}_{\mathcal{H}}^{2} h_{1}, \\
& 0=\mathcal{R}_{\mathcal{H}}^{\prime}+2 \mathcal{I}_{\mathcal{H}} h-2 \mathcal{I}_{\mathcal{H}} \mathcal{R}_{\mathcal{H}} h_{1},
\end{aligned}
$$

correspondingly. This two relations together with the definition of the entropy function $\mathcal{K}_{\mathcal{H}}=\log \mathcal{I}_{\mathcal{H}}-\mathcal{J}_{\mathcal{H}}$ and formula $\mathcal{J}_{\mathcal{H}}^{\prime}=-2 \xi_{\mathcal{H}}^{\prime}+2 h_{1} \mathcal{I}_{\mathcal{H}}$ imply the formulas for $\mathcal{K}_{\mathcal{H}}^{\prime}, \mathcal{I}_{\mathcal{H}}^{\prime} / \mathcal{I}_{\mathcal{H}}$, and $\mathcal{R}_{\mathcal{H}}^{\prime} / \mathcal{I}_{\mathcal{H}}$ in the case where $\mathcal{H}$ is smooth. These formulas in the general case then follow as in the proof of Lemma 2.7 in [1. It remains to show that the spectral measure $\mu_{r}^{d}=w_{r}^{d}(x) d x+\mu_{\mathbf{s}, r}^{d}$ for the Hamiltonian $\mathcal{H}_{r}^{d}: t \mapsto \mathcal{H}^{d}(t+r)$ belongs to $\mathrm{Sz}(\mathbb{R})$ and $\mathcal{K}_{\mathcal{H}^{d}}(r)=\mathcal{K}_{\mathcal{H}}(r)$ for every $r \geqslant 0$. This fact for diagonal Hamiltonians $\mathcal{H}$ is the part of Lemma 2.5 of [1]. However, the proof of this part actually does not uses the diagonal structure of $\mathcal{H}$ and hence works in our situation as well.

\section{REFERENCES}

[1] R. V. Bessonov and S. A. Denisov. A spectral Szegô theorem on the real line. Preprint arXiv:1711.05671, 2017.

[2] M. Christ and A. Kiselev. Scattering and wave operators for one-dimensional Schrödinger operators with slowly decaying nonsmooth potentials. Geom. Funct. Anal., 12(6):1174-1234, 2002.

[3] L. de Branges. Hilbert spaces of entire functions. Prentice-Hall, Inc., Englewood Cliffs, N.J., 1968.

[4] S. A. Denisov. On the existence of wave operators for some Dirac operators with square summable potential. Geom. Funct. Anal., 14(3):529-534, 2004.

[5] S. A. Denisov. Continuous analogs of polynomials orthogonal on the unit circle and Krein systems. IMRS Int. Math. Res. Surv., pages 1-148, 2006, Art. ID 54517.

[6] S. Hassi, H. De Snoo, and H. Winkler. Boundary-value problems for two-dimensional canonical systems. Integral Equations Operator Theory, 36(4):445-479, 2000.

[7] I. S. Kac and M. G. Krein. On the spectral functions of the string. Supplement II to the Russian edition of F.V. Atkinson, Discrete and continuous boundary problems, 1968. English translation: Amer. Math. Soc. Transl., (2) 103 (1974), 19-102.

[8] Sergei Khrushchev. Schur's algorithm, orthogonal polynomials, and convergence of Wall's continued fractions in $L^{2}(T)$. Journal of Approximation Theory, 108(2):161-248, 2001.

[9] M. G. Krein. Continuous analogues of propositions on polynomials orthogonal on the unit circle. Dokl. Akad. Nauk SSSR (N.S.), 105:637-640, 1955.

[10] B. M. Levitan and I. S. Sargsjan. Sturm-Liouville and Dirac operators, volume 59 of Mathematics and its Applications (Soviet Series). Kluwer Academic Publishers Group, Dordrecht, 1991. Translated from the Russian.

[11] Michael Reed and Barry Simon. Methods of modern mathematical physics. III. Academic Press, New York-London, 1979.

[12] R. Romanov. Canonical systems and de Branges spaces. preprint arXiv:1408.6022, 2014.

[13] A. Teplyaev. A note on the theorems of M. G. Krein and L. A. Sakhnovich on continuous analogs of orthogonal polynomials on the circle. J. Funct. Anal., 226(2):257-280, 2005. 
[14] H. Winkler. The inverse spectral problem for canonical systems. Integral Equations Operator Theory, 22(3):360-374, 1995.

BESSONOV@PDMI.RAS.RU

St. Petersburg State University

Universitetskaya nab. 7/9, 199034 St. Petersburg, RUSSiA

St. Petersburg Department of Steklov Mathematical Institute of RAS

Fontanka 27, 191023 St.Petersburg, RUSSIA 